MATHEMATICS OF COMPUTATION

Volume 79, Number 270, April 2010, Pages 1019-1045

S 0025-5718(09)02301-1

Article electronically published on September 24, 2009

\title{
DISK-LIKE TILES AND SELF-AFFINE CURVES WITH NONCOLLINEAR DIGITS
}

\author{
IBRAHIM KIRAT
}

\begin{abstract}
Let $A \in M_{n}(\mathbb{Z})$ be an expanding matrix, $D \subset \mathbb{Z}^{n}$ a digit set and $T=T(A, D)$ the associated self-affine set. It has been asked by Gröchenig and Haas (1994) that given any expanding matrix $A \in M_{2}(\mathbb{Z})$, whether there exists a digit set such that $T$ is a connected or disk-like (i.e., homeomorphic to the closed unit disk) tile. With regard to this question, collinear digit sets have been studied in the literature. In this paper, we consider noncollinear digit sets and show the existence of a noncollinear digit set corresponding to each expanding matrix such that $T$ is a connected tile. Moreover, for such digit sets, we give necessary and sufficient conditions for $T$ to be a disk-like tile.
\end{abstract}

\section{INTRODUCTION}

Let $M_{n}(\mathbb{Z})$ be the set of $n \times n$ matrices with entries in $\mathbb{Z}$ and let $A \in M_{n}(\mathbb{Z})$ be an expanding matrix; i.e., all its eigenvalues have moduli $>1$. Let $D=\left\{d_{1}, \cdots, d_{q}\right\} \subset$ $\mathbb{R}^{n}$ be a set of $q$ distinct vectors, called a digit set. The affine maps $S_{j}(x)=$ $A^{-1}\left(x+d_{j}\right), 1 \leq j \leq q$, are contractions under a suitable norm on $\mathbb{R}^{n}$ [11; thus there is a unique nonempty compact set $T$ satisfying $T=\bigcup_{j=1}^{q} S_{j}(T)$ which can be explicitly given by

$$
T:=T(A, D)=\left\{\sum_{k=1}^{\infty} A^{-k} d_{j_{k}}: d_{j_{k}} \in D\right\} .
$$

In other words, $T$ is the attractor of the iterated function system $\left\{S_{j}\right\}_{j=1}^{q}$, which is a set of radix expansions, and called a self-affine set. If $|\operatorname{det}(A)|=q$ and $T$ has nonempty interior, then it is called a self-affine tile. A connected self-affine set is locally connected, and so it is a curve by the Hahn-Mazurkiewicz Theorem. Therefore, we call a connected self-affine set a self-affine curve. In this paper, we will be concerned with integer digit sets, i.e., $D \subset \mathbb{Z}^{n}$.

There is a growing literature on the formalization and representation of topological questions; see [4] for a survey of the field. One of the interesting aspects of the self-affine sets is the connectedness. This property is important in computer vision and remote sensing [8, 19. As explained in 2, there is some motivation for studying connected self-affine tiles because they are related to number systems, wavelets, and torus maps. Recently, there have been intensive investigations on the

Received by the editor November 7, 2007 and, in revised form, July 25, 2008.

2000 Mathematics Subject Classification. Primary 52C20, 05B45; Secondary 37C70.

Key words and phrases. Self-affine tiles, disk-like tiles, connectedness.

(C)2009 American Mathematical Society 
topic by Akiyama and Thuswaldner [1, 15, Ngai and Tang [17, 18] and Luo et al. [15, 16.

Usually, the boundary of a tile has a complicated geometry and is of considerable interest. We note that once we get a connected tile $T$ for dimensions $n \geq 2$, we automatically have a connected boundary (see [15]), which is a curve for $n=2$ (of course, that is the case if $T$ is disk-like) by the work of Luo in [14. This is another aspect of connected tiles.

It was conjectured and partially solved in [6 that for any expanding matrix $A \in M_{2}(\mathbb{Z})$, there exists a digit set $D$ such that $T(A, D)$ is a connected tile. This problem was solved in 9]. However, in [9, 10, we were mainly interested in collinear digits (i.e., $D \subset \mathbb{Z}^{n}$ is a subset of a straight line). Little is known about the connectedness of self-affine sets with non-collinear digits.

The study of disklikeness (i.e., being homeomorphic to the closed unit disk) is interesting partly because, in many cases, "almost all self-affine tiles have holes or disconnected interiors" as mentioned in [3]. Bandt and Gelbrich studied the disklikeness problem for the cases $|\operatorname{det}(A)|=2,3$ and showed that for $\operatorname{det}(A)=-3$ and $\operatorname{tr}(A)= \pm 1$ ( $\operatorname{tr}$ denotes the trace), there is no digit set $D$ such that $T(A, D)$ is disk-like [2].

Leung and Lau also considered collinear digits 13 . They assumed that $D$ is a consecutive collinear digit set in $\mathbb{Z}^{2}, T(A, D)$ is a self-affine tile, and showed that $T(A, D)$ is disk-like if and only if $2|p| \leq|q+2|$, where $f(x)=x^{2}+p x+q$ is the characteristic polynomial of $A$. But collinear digits may not give tiles since for $A=$ $q I$, where $|q| \geq 2$ and $I$ is the identity matrix, $T(A, D)$ is not a tile for any collinear digit set $D=\left\{0, d_{1} v, \cdots, d_{|q|^{2}-1} v\right\}$ with $v \in \mathbb{R}^{2} \backslash\{0\}, d_{1}, \cdots, d_{|q|^{2}-1} \in \mathbb{N}$. Hence, there is no disk-like tile to study in this case, but the analog of the connectedness criterion in $\mathbb{R}[9]$ holds:

$T(A, D)$ is connected if and only if, up to a translation, $D=a\left\{0, v, 2 v, \ldots,\left(|q|^{2}-\right.\right.$ 1) $v\}$ for some $a>0$.

Less is known about the disklikeness when the digits are noncollinear. In regard to this problem, Gmainer and Thuswaldner 5 study the disklikeness of a class of tiles with noncollinear digits arising from polyominoes. The study of such digits is more delicate than collinear digits. Our work will show that a small change in a collinear digit set causes considerable complications in the study of disklikeness. Clearly, if $D$ consists of two digits, $D$ is a collinear digit set. Hence we only need to study $D$ with more than two digits. The work presented here is inspired by the results in the papers [9, 13. In this paper, we will be concerned with self-affine sets with noncollinear digit sets and prove the following existence and characterization results.

Theorem 1.1. For any expanding matrix $A \in M_{2}(\mathbb{Z})$ with irreducible characteristic polynomial $x^{2}+p x+q,|q| \geq 3$, there is a noncollinear digit set $D$ (in fact, there exist infinitely many) so that $T(A, D)$ is a connected tile and

(i) when $q$ is even, $T(A, D)$ is disk-like if and only if $2|p| \leq|q+2|$,

(ii) when $q>0$ is odd, $T(A, D)$ is disk-like if and only if $2|p| \leq|q+3|$ and $p \neq \pm 1$,

(iii) when $q<0$ is odd, $T(A, D)$ is disk-like if and only if $2|p| \leq|q+3|$.

Theorem 1.2. For any expanding matrix $A \in M_{2}(\mathbb{Z})$ with reducible characteristic polynomial $x^{2}+p x+q$, there exists a noncollinear digit set such that the associated self-affine set is a disk-like tile. (See Figure 5 .) 
Also, the Lebesgue measures of the tiles in the above theorems are immediately obtained from the proofs. It follows from Theorem 1.1 and Theorem 1.2 that there always exist noncollinear digit sets $D$ for $p=0$ so that $T$ is a disk-like tile. Our study is divided into two parts:

I) We prove Theorem 1.1 by reducing the problem to companion matrices, and then finding desired digit sets for such matrices.

II) We prove Theorem 1.2 by reducing the problem to a matrix of the form $\left[\begin{array}{cc}m & 0 \\ t & n\end{array}\right]$, and then giving a digit set with the required properties.

As for the organization of the paper, we give some preparatory results in Section 2. We prove Theorem 1.1 in Section 3 as a combination of several propositions. Theorem 1.2 is proved in Section 4 In the last section, we list the digit sets for each case and summarize the results of this work in more detail as a table in the appendix. We also have several figures of finite point-set approximations to connected tiles, which are generated by using MATLAB. The different shades of gray in those figures correspond to the $q$ pieces $A^{-1}\left(T+d_{j}\right), 1 \leq j \leq q$, of the self-affine set $T$.

\section{Preliminaries}

In this section, we list the results that will be frequently used in the following sections. We first give the following criterion of the connectedness. It was first proved in [7] and later rediscovered in [9] independently.

Theorem 2.1 ([7], 9]). Let $T$ be an attractor defined by $A \in M_{n}(\mathbb{Z})$ and a digit set $D \subset \mathbb{R}^{n}$. Define

$$
\Sigma=\left\{\left(d_{i}, d_{j}\right): d_{i}, d_{j} \in D \text { and }\left(T+d_{i}\right) \cap\left(T+d_{j}\right) \neq \emptyset\right\} .
$$

Then $T$ is connected if and only if for any pair of $d_{i}, d_{j} \in D$, there exists a finite set $\left\{d_{j_{1}}, \cdots, d_{j_{k}}\right\} \subset D$ such that $d_{j_{1}}=d_{i}, d_{j_{k}}=d_{j}$ and $\left(d_{j_{l}}, d_{j_{l+1}}\right) \in \Sigma$ for $1 \leq l \leq k-1$.

It is easy to see that $\left(d_{i}, d_{j}\right) \in \Sigma$ if and only if

$$
d_{i}-d_{j}=\sum_{k=1}^{\infty} A^{-k} v_{k}, \quad v_{k} \in D-D .
$$

However, it is not clear how to check whether $\left(d_{i}, d_{j}\right) \in \Sigma$ or not.

We continue with the definitions. If $T$ is a tile, then there exists a subset $\mathcal{J} \subseteq \mathbb{Z}^{n}$ such that

$$
T+\mathcal{J}=\mathbb{R}^{n} \quad \text { and } \quad(T+t)^{o} \cap\left(T+t^{\prime}\right)^{o}=\emptyset, \quad t \neq t^{\prime}, t, t^{\prime} \in \mathcal{J}
$$

(see e.g., [12]). $\mathcal{J}$ is called a tiling set; $T+\mathcal{J}$ is called a tiling of $\mathbb{R}^{n}$ and a lattice tiling if $\mathcal{J}$ is a lattice. When $\mathcal{J}=\mathbb{Z}^{n}$, the Lebesgue measure of $T, \mu(T)$, is 1 [12], and $T$ is called a $\mathbb{Z}^{n}$-tile or a Haar tile (because of its relation to Haar wavelets). If $v \in \mathbb{Z}^{n} \backslash\{0\}$ and $(T+v) \cap T \neq \emptyset$, then $(T+v)$ is called a neighbor of $T$.

Theorem $2.2\left([2,[3])\right.$. Let $T$ be a disk-like tile and $T+\mathcal{J}$ a lattice tiling of $\mathbb{R}^{2}$. Then one of the following holds.

(i) $T$ has exactly 6 neighbors $T \pm \ell_{1}, T \pm \ell_{2}, T \pm\left(\ell_{1}+\ell_{2}\right)$ for some $\ell_{1}, \ell_{2} \in \mathcal{J}$ and $\ell_{1} \mathbb{Z}+\ell_{2} \mathbb{Z}=\mathcal{J}$.

(ii) $T$ has exactly 8 neighbors $T \pm \ell_{1}, T \pm \ell_{2}, T \pm\left(\ell_{1}+\ell_{2}\right), T \pm\left(\ell_{1}-\ell_{2}\right)$ for some $\ell_{1}, \ell_{2} \in \mathcal{J}$, and $\ell_{1} \mathbb{Z}+\ell_{2} \mathbb{Z}=\mathcal{J}$. 
Theorem $2.3(\underline{3})$. Let $T$ be a $\mathbb{Z}^{2}$-tile with not more than six neighbors. Then $T$ is disk-like if and only if $T$ is connected.

The characterizations in Theorem 2.2 and Theorem 2.3 are sharp. In order to use these theorems, it is crucial to obtain the neighbors. There are also graph-theoretic algorithms to determine the neighbors of $T$ [20, 21]. It is mentioned in [20] that the algorithm there is more suitable for a large class of tiles and faster than the one in 21. Each of our proofs uses a different matrix (or matrices) and mostly a different class (or classes) of digit sets. The implementation of those algorithms in [20, 21] for each proof and each digit set here does not look much easier than our calculations as can be seen from [5]. On the other hand, the ad hoc methods used in this paper to determine the neighbors of the tiles directly give explicit infinite digit expansions of a few boundary points for each tile.

We will also need the following, which can be found in [9.

Proposition 2.4. Let $A \in M_{n}(\mathbb{Z})$ be an expanding matrix and let $D_{1}, D_{2}$ be two digit sets with $D_{2}=x_{0}+D_{1}$ for some $x_{0} \in \mathbb{R}^{n}$. Then the two attractors $T_{1}$ and $T_{2}$ corresponding to $\left(A, D_{1}\right)$ and $\left(A, D_{2}\right)$ are related by

$$
T_{2}=T_{1}+(A-I)^{-1} x_{0} .
$$

Proposition 2.5. Let $A \in M_{n}(\mathbb{Z})$ be an expanding matrix and $D \subseteq \mathbb{R}^{n}$ be a digit set. Suppose that there exists a vector $v \in \mathbb{R}^{n}$ such that $D=v-D$. Then

$$
T(-A, D)=T(A, D)-\left(A-A^{-1}\right)^{-1} v .
$$

Lemma 2.6. Let $f(x)=x^{2}+a x \pm q$, where $a \in \mathbb{Z}, 1 \neq q \in \mathbb{N}$. Then $f(x)$ is expanding if and only if $|a| \leq q$ for $f(0)=q$, and $|a| \leq q-2$ for $f(0)=-q$.

Lemma 2.7. Let $f(x)$ and $\tilde{f}(x)$ denote the characteristic polynomials of $A,-A \in$ $M_{n}(\mathbb{Z})$, respectively. Then $\tilde{f}(x)=(-1)^{n} f(-x)$.

\section{The irReducible-CharaCtERISTIC-POLYNOMIAL CASE}

In this section, we will assume that all characteristic polynomials $x^{2}+p x+q$ are irreducible and $|q| \geq 3$. We will need the following results.

Proposition 3.1. For any expanding matrix $A \in M_{n}(\mathbb{Z})$ with irreducible characteristic polynomial $f(x), \mathcal{B}=\left\{v, A v, \cdots A^{n-1} v\right\}$ is a linearly independent set for any $v \in \mathbb{Z}^{n} \backslash\{0\}$, and the matrix representation of $A$ in basis $\mathcal{B}$ is the companion matrix $C$ of $f(x) ; C$ has 1 's below the diagonal, the negatives of the coefficients of $f(x)$ in the last column, and zeroes at all other entries.

Proof. The proof follows from the proof of Theorem 3.1 in $[9]$.

By Proposition 3.1, it is sufficient to study companion matrices for our purposes. A digit set $D$ is said to be a primitive digit set if the lattice $\mathcal{J}$ generated by $\left\{A^{i} D\right\}_{i=0}^{n-1}$ is $\mathbb{Z}^{n}$. D is called a complete residue system $(\bmod A)$ or just a complete digit set if it is a complete set of coset representatives of the additive group $\mathbb{Z}^{n} / A \mathbb{Z}^{n}$. For future use, we state the following fact so that Theorem 2.2 and Theorem 2.3 apply.

Theorem $3.2([12])$. Let $T=T(A, D)$ be a tile generated by a primitive complete digit set $D$ and an expanding matrix $A \in M_{n}(\mathbb{Z})$ with irreducible characteristic polynomial over $\mathbb{Z}$. Then $\mu(T)=1$ and $T$ is a Haar tile. 

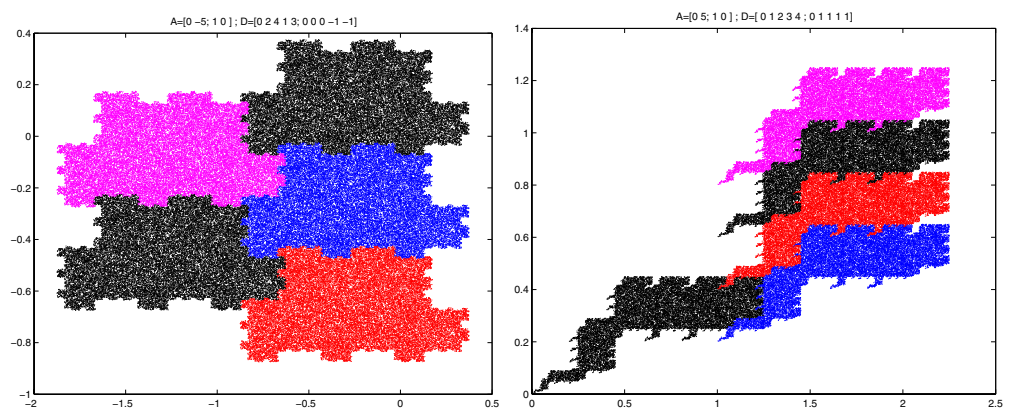

Figure 1. Disk-like tiles of Proposition 3.4

The following trivial lemma will be used together with Proposition 2.4 or Proposition 2.5 .

Lemma 3.3. Let $T$ be a tile and $v, x \in \mathbb{R}^{n}$. Then $T \cap(T+v) \neq \emptyset$ if and only if $(T+x) \cap(T+x+v) \neq \emptyset$.

For simplicity, we will denote an element $\sum_{k=1}^{\infty} A^{-k} d_{j_{k}} \in T, d_{j_{k}} \in D$, by the sequence $d_{j_{1}} d_{j_{2}} d_{j_{3}} \ldots$. As a special case, a periodic sequence will be a sequence of the form $d_{j_{1}} \ldots d_{j_{m}} d_{j_{m+1}} \ldots d_{j_{m+s}} d_{j_{m+1}} \ldots d_{j_{m+s}} \ldots=d_{j_{1}} \ldots d_{j_{m}} \overline{d_{j_{m+1}} \ldots d_{j_{m+s}}}$, i.e., the block of digits $d_{j_{m+1}}, d_{j_{m+2}}, \ldots, d_{j_{m+s}}$ is repeated indefinitely. The proof of Theorem 1.1 will follow from the series of propositions below.

Proposition 3.4. For any expanding matrix $A \in M_{2}(\mathbb{Z})$ with characteristic polynomial $f(x)=x^{2} \pm q, q>2$, there exists a noncollinear digit set $D$ so that $T(A, D)$ is disk-like. (See Figure 1.)

Proof. (A) $f(x)=x^{2}-q$ : Without loss of generality (abbreviated as WLOG), we only consider the companion matrices $A=\left[\begin{array}{ll}0 & q \\ 1 & 0\end{array}\right]$ with characteristic polynomial $x^{2}-q$ (see Proposition 3.1) and let $D=\left\{\left[\begin{array}{l}0 \\ 0\end{array}\right],\left[\begin{array}{l}k \\ 1\end{array}\right]: 1 \leq k \leq q-1\right\}$ such that

$$
D=\left\{d_{1}=\left[\begin{array}{l}
0 \\
0
\end{array}\right], d_{2}=\left[\begin{array}{l}
1 \\
1
\end{array}\right], \cdots, d_{q-1}=\left[\begin{array}{c}
q-2 \\
1
\end{array}\right], d_{q}=\left[\begin{array}{c}
q-1 \\
1
\end{array}\right]\right\}
$$

for $q \geq 3$. We want to show that $T=T(A, D)$ is disk-like. We first note that $\mu(T)=1$ by Theorem 3.2 since $f(x)$ is irreducible by our assumption and $D$ is a primitive complete digit set. Our plan is to show that $T \pm\left[\begin{array}{l}2 \\ 1\end{array}\right], T \pm\left[\begin{array}{l}1 \\ 0\end{array}\right]$, $T \pm\left[\begin{array}{l}1 \\ 1\end{array}\right]$ are the only neighbors of $T$, and thus Theorem 2.1 and Theorem 2.3 imply that $T$ is disk-like. It is clear that if $T+v$ is a neighbor of $T$, then $T-v$ is also a neighbor. Hence it is enough to show that $T+\left[\begin{array}{l}2 \\ 1\end{array}\right], T+\left[\begin{array}{l}1 \\ 0\end{array}\right], T+\left[\begin{array}{l}1 \\ 1\end{array}\right]$ are the only neighbors of $T$ for the disklikeness.

We now begin to determine the neighbors of $T$. We will leave the verification of all necessary matrix computations to the reader. We note that

$$
\overline{d_{q-1}}=\left[\begin{array}{l}
2 \\
1
\end{array}\right], \quad \overline{d_{2} d_{1}}-\overline{d_{1} d_{2}}=\left[\begin{array}{l}
1 \\
0
\end{array}\right], \quad \overline{d_{q}}-\overline{d_{2} d_{1}}=\left[\begin{array}{l}
1 \\
1
\end{array}\right] .
$$

Hence $T+\left[\begin{array}{l}2 \\ 1\end{array}\right], T+\left[\begin{array}{l}1 \\ 0\end{array}\right], T+\left[\begin{array}{l}1 \\ 1\end{array}\right]$ are neighbors of $T$. 
We show that there are no other neighbors. To this end, we notice that all of the entries of $A^{k}$ are nonnegative. Hence, for the above digit sets, it is easy to see that $\left[\begin{array}{l}x \\ y\end{array}\right] \in T-T$ implies that

$$
\left[\begin{array}{l}
|x| \\
|y|
\end{array}\right] \leq \sum_{k=1}^{\infty} A^{-k}\left[\begin{array}{c}
q-1 \\
1
\end{array}\right]=(A-I)^{-1}\left[\begin{array}{c}
q-1 \\
1
\end{array}\right]=\left[\begin{array}{c}
2+\frac{1}{q-1} \\
1+\frac{1}{q-1}
\end{array}\right]
$$

so that $|x| \leq 2$ and $|y| \leq 1$ for integers $x, y$. We need to show that $\left[\begin{array}{c}2 \\ -1\end{array}\right],\left[\begin{array}{c}-1 \\ 1\end{array}\right]$, $\left[\begin{array}{l}0 \\ 1\end{array}\right]$ and $\left[\begin{array}{l}2 \\ 0\end{array}\right]$ are not in $T-T$. Recall that

$$
(A-I)^{-1}\left[\begin{array}{c}
q-1 \\
1
\end{array}\right]=\left[\begin{array}{c}
2+\frac{1}{q-1} \\
1+\frac{1}{q-1}
\end{array}\right], \quad(A-I)^{-1}\left[\begin{array}{c}
q-2 \\
1
\end{array}\right]=\left[\begin{array}{l}
2 \\
1
\end{array}\right] .
$$

From these we see that $\left[\begin{array}{l}x \\ y\end{array}\right] \in T-T$ and $x \geq 2$ imply that all the digits $d$ in the decimal expansion of $\left[\begin{array}{l}x \\ y\end{array}\right]$ must satisfy $d \geq\left[\begin{array}{c}q-2 \\ 1\end{array}\right]$. This gives $y \geq 1$. Thus $\left[\begin{array}{c}2 \\ -1\end{array}\right],\left[\begin{array}{l}2 \\ 0\end{array}\right]$ cannot be in $T-T$.

On the other hand, $\left[\begin{array}{l}x \\ y\end{array}\right] \in T-T$ and $y \geq 1$ also imply that all the digits $d$ in the decimal expansion of $\left[\begin{array}{l}x \\ y\end{array}\right]$ must satisfy $d \geq\left[\begin{array}{c}q-2 \\ 1\end{array}\right]$. So we must have $x \geq 2$. Then $\left[\begin{array}{c}-1 \\ 1\end{array}\right],\left[\begin{array}{l}0 \\ 1\end{array}\right]$ cannot be in $T-T$ either.

(B) $f(x)=x^{2}+q$ :

(i) We now consider the companion matrices $A$ with characteristic polynomial $x^{2}+q$, where $q>2$ is even and $D=\left\{\left[\begin{array}{c}2 k \\ 0\end{array}\right],\left[\begin{array}{c}2 k \\ -1\end{array}\right]: 0 \leq k \leq \frac{q-2}{2}\right\}$. Then $A D=\left\{\left[\begin{array}{c}0 \\ 2 k\end{array}\right],\left[\begin{array}{c}q \\ 2 k\end{array}\right]: 0 \leq k \leq \frac{q-2}{2}\right\}$ and

$$
\begin{aligned}
D+A D & =\left\{\left[\begin{array}{c}
2 k \\
2 l
\end{array}\right],\left[\begin{array}{c}
2 k \\
2 l-1
\end{array}\right],\left[\begin{array}{c}
2 k+q \\
2 l
\end{array}\right],\left[\begin{array}{c}
2 k+q \\
2 l-1
\end{array}\right]: 0 \leq k, l \leq \frac{q-2}{2}\right\} \\
& =\left\{\left[\begin{array}{c}
2 k \\
2 l
\end{array}\right],\left[\begin{array}{c}
2 k \\
2 l-1
\end{array}\right]: 0 \leq k \leq q-1,0 \leq l \leq \frac{q-2}{2}\right\} .
\end{aligned}
$$

Set

$$
\begin{aligned}
D^{\prime}=\left[\begin{array}{l}
0 \\
1
\end{array}\right]+D+A D & =\left\{\left[\begin{array}{c}
2 k \\
2 l
\end{array}\right],\left[\begin{array}{c}
2 k \\
2 l+1
\end{array}\right]: 0 \leq k \leq q-1,0 \leq l \leq \frac{q-2}{2}\right\} \\
& =\left\{\left[\begin{array}{c}
2 k \\
l
\end{array}\right]: 0 \leq k, l \leq q-1\right\} .
\end{aligned}
$$

Then $T(A, D)=T\left(A^{2}, D^{\prime}\right)-\left(A^{2}-I\right)^{-1}\left[\begin{array}{l}0 \\ 1\end{array}\right]$ by Proposition 2.4. Note that $A^{2}=$ $-q I=: \tilde{A}, \sum_{k=1}^{\infty} \tilde{A}^{-2 k}=\left(\tilde{A}^{2}-I\right)^{-1}=\frac{1}{q^{2}-1} I$, and $\sum_{k=1}^{\infty} \tilde{A}^{-(2 k-1)}=\tilde{A}\left(\tilde{A}^{2}-I\right)^{-1}=$ $\frac{-q}{q^{2}-1} I$. Then $\left[\begin{array}{l}x \\ y\end{array}\right] \in T\left(A^{2}, D^{\prime}\right)$ implies that $-2 q \frac{q-1}{q^{2}-1} \leq x \leq 2 \frac{q-1}{q^{2}-1}, \quad-q \frac{q-1}{q^{2}-1} \leq y \leq$ $\frac{q-1}{q^{2}-1}$. Therefore, $T\left(A^{2}, D^{\prime}\right)=2\left[\frac{-q}{q+1}, \frac{1}{q+1}\right] \times\left[\frac{-q}{q+1}, \frac{1}{q+1}\right]$ and so

$$
T(A, D)=T\left(A^{2}, D^{\prime}\right)-\left(A^{2}-I\right)^{-1}\left[\begin{array}{l}
0 \\
1
\end{array}\right]=2\left[\frac{-q}{q+1}, \frac{1}{q+1}\right] \times\left[\frac{-(q-1)}{q+1}, \frac{2}{q+1}\right] .
$$

(ii) We next consider the companion matrices $A$ with characteristic polynomial $x^{2}+q$, where $q>2$ is odd, and $D=\left\{\left[\begin{array}{c}2 k \\ 0\end{array}\right],\left[\begin{array}{c}2 l+1 \\ -1\end{array}\right]: 0 \leq k \leq \frac{q-1}{2}, 0 \leq l \leq\right.$ $\left.\frac{q-3}{2}\right\}$. Note that $D$ is not a primitive digit set. Therefore, we transform it to a 
primitive complete digit set so that Theorem 3.2 applies. For our purposes, we let $B^{-1}=\left[\begin{array}{cc}\frac{1}{2} & \frac{1}{2} \\ 0 & -1\end{array}\right]$. Then $B^{-1} A B=\left[\begin{array}{cc}1 & \frac{q+1}{2} \\ -2 & \frac{-1}{-1}\end{array}\right]$, and

$$
B^{-1} D=\left\{\left[\begin{array}{l}
0 \\
0
\end{array}\right],\left[\begin{array}{l}
1 \\
0
\end{array}\right],\left[\begin{array}{l}
2 \\
0
\end{array}\right], \cdots,\left[\begin{array}{c}
\frac{q-1}{2} \\
0
\end{array}\right],\left[\begin{array}{l}
0 \\
1
\end{array}\right],\left[\begin{array}{l}
1 \\
1
\end{array}\right],\left[\begin{array}{l}
2 \\
1
\end{array}\right], \cdots,\left[\frac{q-3}{2}\right]\right\} .
$$

Since $T\left(B^{-1} A B, B^{-1} D\right)=B^{-1} T(A, D)$, we may assume that $A=\left[\begin{array}{cc}1 & \frac{q+1}{2} \\ -2 & -1\end{array}\right]$ and

$$
D=\left\{d_{1}=\left[\begin{array}{l}
0 \\
0
\end{array}\right], d_{2}=\left[\begin{array}{l}
1 \\
0
\end{array}\right], \cdots, d_{\frac{q+1}{2}}=\left[\frac{q-1}{2}\right], d_{\frac{q+3}{2}}=\left[\begin{array}{l}
0 \\
1
\end{array}\right], \cdots,\left[\frac{q-3}{2}\right]\right\}
$$

in the rest of the proof. We now show that $T \pm\left[\begin{array}{l}1 \\ 0\end{array}\right], T \pm\left[\begin{array}{c}1 \\ -1\end{array}\right], T \pm\left[\begin{array}{l}0 \\ 1\end{array}\right]$ are neighbors of $T$. Notice that for $k \in \mathbb{N}$, we have

$$
A^{-2 k}=\left(\frac{-1}{q}\right)^{k} I=\left\{\begin{array}{ll}
\frac{1}{q^{k}} I & \text { if } k \text { is even, } \\
\frac{-1}{q^{k}} I & \text { if } k \text { is odd, }
\end{array} \quad A^{-(2 k-1)}=\left\{\begin{array}{l}
\frac{1}{q^{k}} A \text { if } k \text { is even, } \\
\frac{-1}{q^{k}} A \text { if } k \text { is odd. }
\end{array}\right.\right.
$$

$T \pm\left[\begin{array}{c}1 \\ -1\end{array}\right]$ is a neighbor of $T: \overline{d_{1} d_{1} d_{\frac{q+1}{2}} d_{\frac{q+1}{2}}}-\overline{d_{\frac{q+1}{2}} d_{\frac{q+1}{2}} d_{1} d_{1}}=\left[\begin{array}{c}1 \\ -1\end{array}\right] \in T-T$.

$T \pm\left[\begin{array}{l}0 \\ 1\end{array}\right]$ is a neighbor of $T: \overline{d_{\frac{q+1}{2}} d_{1} d_{1} d_{\frac{q+1}{2}}}-\overline{d_{1} d_{\frac{q+1}{2}} d_{\frac{q+1}{2}} d_{1}}=d_{\frac{q+3}{2}} \in T-T$.

$T \pm\left[\begin{array}{l}1 \\ 0\end{array}\right]$ is a neighbor of $T$ :

$$
d_{\frac{q+3}{2}} d_{\frac{q+1}{2}} \overline{d_{\frac{q+1}{2}} d_{1} d_{1} d_{\frac{q+1}{2}}}-d_{1} d_{1} \overline{d_{1} d_{\frac{q+1}{2}} d_{\frac{q+1}{2}} d_{1}}=-d_{2} \in T-T .
$$

We next show that these are the only neighbors of $T$ and, by Theorem 2.3. $T$ is disk-like. First note that $\left[\begin{array}{l}x \\ y\end{array}\right] \in T-T$ implies that $|x| \leq$ the first entry of

$$
\begin{gathered}
{\left[\begin{array}{c}
\frac{3}{2} \\
\cdot
\end{array}\right]=\sum_{\text {even } k} A^{-2 k}\left[\frac{q-1}{2}\right]-\sum_{\text {odd } k} A^{-2 k}\left[\frac{q-1}{2}\right]+\sum_{\text {even } k} A^{-(2 k-1)}\left[\frac{q-3}{2}\right]} \\
-\sum_{\text {odd } k} A^{-(2 k-1)}\left[\frac{q-3}{\frac{2}{1}}\right]=\left[\begin{array}{c}
\frac{1}{2} \\
0
\end{array}\right]+\frac{1}{q-1} A\left[\begin{array}{c}
\frac{q-3}{2} \\
1
\end{array}\right]=\left[\begin{array}{c}
\frac{1}{2} \\
0
\end{array}\right]+\frac{1}{q-1}\left[\frac{q-3}{2}+\frac{q+1}{2}\right]
\end{gathered}
$$

so that $|x| \leq \frac{3}{2}$. Also $\left[\begin{array}{l}x \\ y\end{array}\right] \in T-T$ implies that $|y| \leq$ the second entry of

$$
\begin{gathered}
\sum_{\text {even } k} A^{-2 k}\left[\begin{array}{l}
0 \\
1
\end{array}\right]-\sum_{\text {odd } k} A^{-2 k}\left[\begin{array}{l}
0 \\
1
\end{array}\right]+\sum_{\text {odd } k} A^{-(2 k-1)}\left[\frac{q-1}{2}\right] \\
-\sum_{\text {even } k} A^{-(2 k-1)}\left[\frac{q-1}{2}\right]=\left[\begin{array}{c}
0 \\
\frac{1}{q-1}
\end{array}\right]+\frac{-1}{q-1} A\left[\frac{q-1}{2}\right]=\left[\begin{array}{c}
0 \\
\frac{1}{q-1}
\end{array}\right] \\
+\frac{-1}{q-1}\left[\begin{array}{c}
\frac{q-1}{2} \\
-(q-1)
\end{array}\right]=\left[\begin{array}{c}
-\frac{1}{2} \\
1+\frac{1}{q-1}
\end{array}\right]
\end{gathered}
$$

so that $|y| \leq 1+\frac{1}{q-1}$. Therefore, $|x| \leq 1$ and $|y| \leq 1$ for integer $\left[\begin{array}{l}x \\ y\end{array}\right] \in T-T$.

We must prove that $T \pm\left[\begin{array}{l}1 \\ 1\end{array}\right]$ are not neighbors of $T$. For this, we only need to note that $y=1$ if and only if we replace the digit $\left[\begin{array}{l}0 \\ 1\end{array}\right]$ by any other digit of the form $\left[\begin{array}{l}\cdot \\ 0\end{array}\right]$ in the above expression. To maximize the first entry of that expression, 

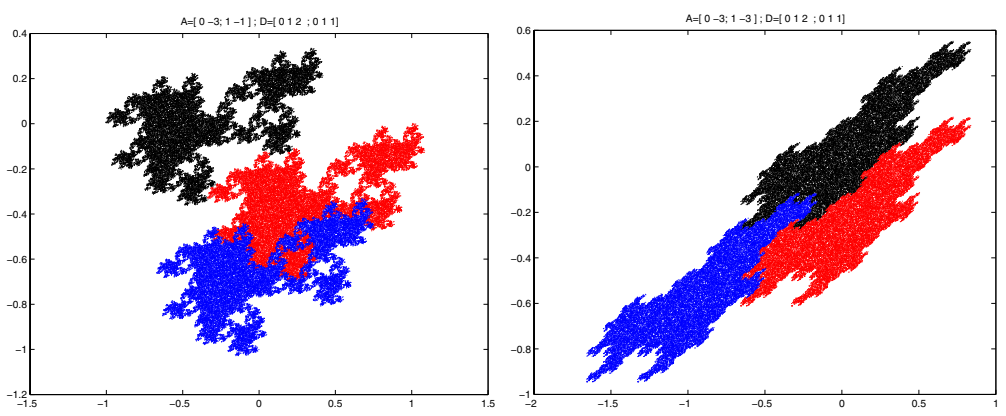

Figure 2. A non-disk-like connected tile (left) and a disk-like tile of Proposition 3.5

we take $\left[\frac{q-1}{2}\right]$ in place of $\left[\begin{array}{l}0 \\ 1\end{array}\right]$. Then

$$
\begin{gathered}
{\left[\begin{array}{l}
0 \\
1
\end{array}\right]=\sum_{\text {even } k} A^{-2 k}\left[\frac{q-1}{2}\right]-\sum_{\text {odd } k} A^{-2 k}\left[\frac{\frac{q-1}{2}}{0}\right]+\sum_{\text {odd } k} A^{-2(k-1)}\left[\frac{q-1}{2}\right]} \\
-\sum_{\text {even } k} A^{-2(k-1)}\left[\frac{q-1}{2}\right]=\left[\begin{array}{c}
\frac{1}{2} \\
0
\end{array}\right]+\frac{-1}{q-1} A\left[\frac{q-1}{2}\right]=\left[\begin{array}{c}
\frac{1}{2} \\
0
\end{array}\right]+\frac{-1}{q-1}\left[\begin{array}{c}
\frac{q-1}{2} \\
-(q-1)
\end{array}\right] .
\end{gathered}
$$

This shows that $x \leq 0$, and so $x$ cannot be 1 when $\left[\begin{array}{l}x \\ 1\end{array}\right] \in T-T$.

In the following, we will write 0 for the zero matrix.

Proposition 3.5. For any expanding matrix $A \in M_{2}(\mathbb{Z})$ with characteristic polynomial $x^{2}+p x+q$, where $q$ is odd and $p, q>0$, there exists a noncollinear digit set $D$ so that $T(A, D)$ is a connected tile and is disk-like if and only if $2 p \leq q+3$ and $p \neq 1$. (See Figure 2.)

Proof. Let $A$ be the companion matrix of $x^{2}+p x+q$ and let

$$
D=\left\{\left[\begin{array}{l}
0 \\
0
\end{array}\right],\left[\begin{array}{l}
k \\
1
\end{array}\right]: 1 \leq k \leq q-1\right\} .
$$

In order to obtain neighbors of $T$, WLOG, we can use translates of $D$ by Proposition 2.4 and Lemma 3.3

(A) Because the proof in (B) below doesn't work for the case $p=1,2$ (namely, $\left[\begin{array}{c}p-2 \\ 1\end{array}\right]$ and $\left[\begin{array}{c}p-1 \\ 1\end{array}\right]$ are not in the last two translated digit sets considered there), we need to study that case separately. For $p=1$, it follows from Table 1 that $T \pm\left[\begin{array}{l}1 \\ 0\end{array}\right], T \pm\left[\begin{array}{c}p-1 \\ 1\end{array}\right]\left(=\left[\begin{array}{l}0 \\ 1\end{array}\right]\right), T \pm\left[\begin{array}{c}p-2 \\ -1\end{array}\right]\left(=\left[\begin{array}{l}-1 \\ -1\end{array}\right]\right), T \pm\left[\begin{array}{c}p-2 \\ 1\end{array}\right]\left(=\left[\begin{array}{c}-1 \\ 1\end{array}\right]\right)$ are neighbors of $T$.

For $p=2$, the same table shows that $T \pm\left[\begin{array}{l}1 \\ 0\end{array}\right], T \pm\left[\begin{array}{c}p-1 \\ 1\end{array}\right]\left(=\left[\begin{array}{l}1 \\ 1\end{array}\right]\right), T \pm$ $\left[\begin{array}{c}p-2 \\ 1\end{array}\right]\left(=\left[\begin{array}{l}0 \\ 1\end{array}\right]\right)$ are neighbors of $T$. By Theorem 2.1, $T$ is connected for $p=1,2$.

(B) For $p>2$, Table 1 shows that $T \pm\left[\begin{array}{l}1 \\ 0\end{array}\right], T \pm\left[\begin{array}{c}p-1 \\ 1\end{array}\right], T \pm\left[\begin{array}{c}p-2 \\ 1\end{array}\right]$ are neighbors of $T$ again. As before, we conclude that $T$ is connected.

(C) When $2 p>q+3$ or $p=1, T$ is not disk-like: The neighbors for $p=1$ obtained in (A) are in the form of Theorem 2.2 (ii). For $p=1$, we see from Table 1 
TABLE 1

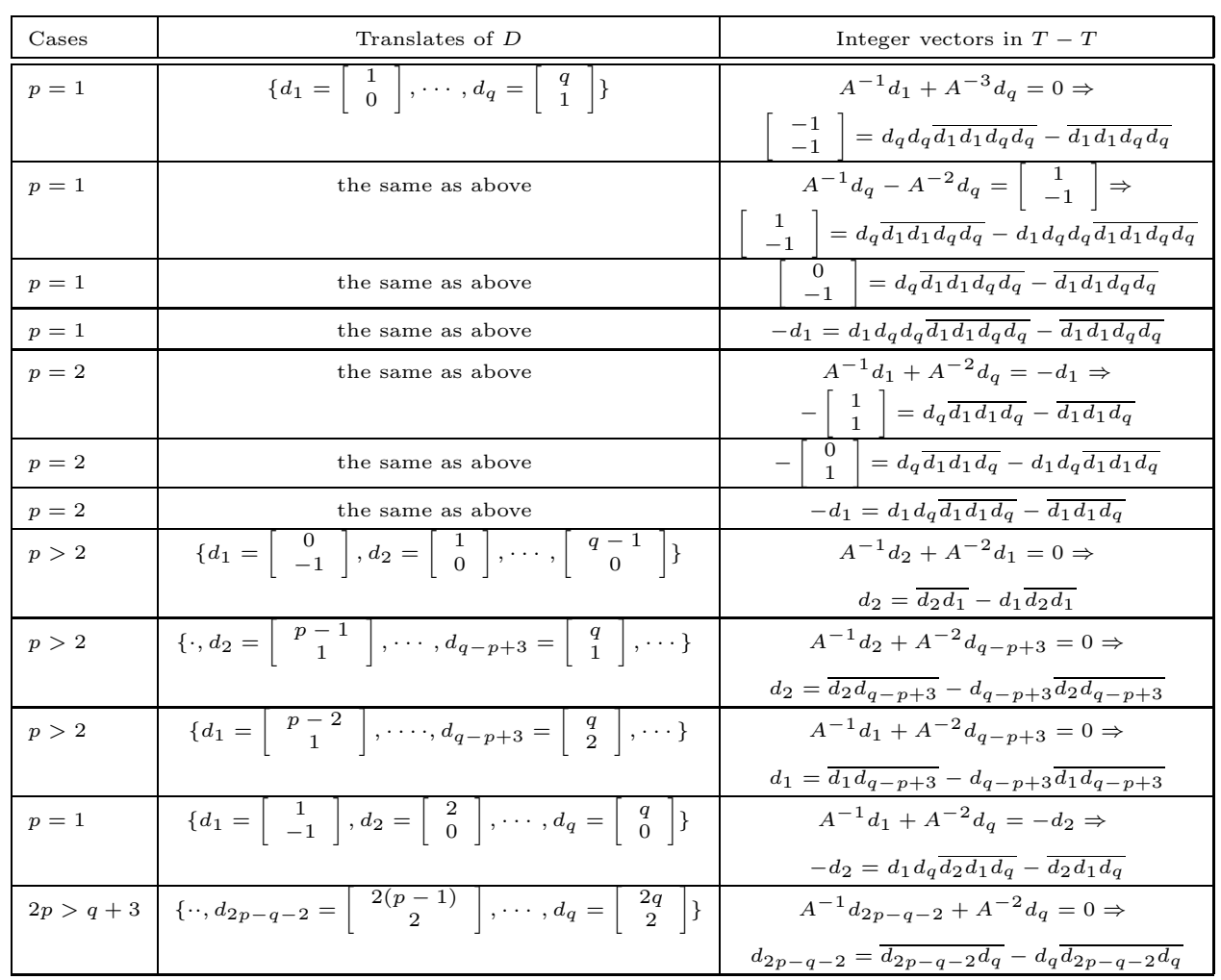

that $T \pm\left[\begin{array}{l}2 \\ 0\end{array}\right]$ is the fifth pair of neighbors of $T$ so that $T$ is not disk-like by Theorem 2.2 and (A).

We now assume that $2 p>q+3$. Note that this inequality implies that $2(p-1) \geq$ $q+2$. Then the last line of Table 1 says that $T+d_{2 p-q-2}$ is another neighbor of $T$. Then, by Theorem 2.2 and (B) in this proof, $T$ is not disk-like.

(D) When $2 p \leq q+3$ and $p \neq 1$, there are no other neighbors: Let $v=\left[\begin{array}{l}1 \\ 0\end{array}\right]$. Then we can write $D$ as $D=\left\{\left[\begin{array}{l}0 \\ 0\end{array}\right], b_{k} v+A v: 1 \leq b_{k} \leq q-1\right\}$. We note that any nonzero integer vector $d \in T-T$ has the form $d=\sum_{k=1}^{\infty} A^{-k}\left(\epsilon_{k}^{\prime} A v\right)+\sum_{k=1}^{\infty} A^{-k} b_{k} v$, where $\epsilon_{k}^{\prime}=0, \pm 1,\left|b_{k}\right| \leq q-1$. Now we want to see the contribution of $\gamma v+\delta A v:=$ $\sum_{k=2}^{\infty} A^{-(k-1)} \epsilon_{k}^{\prime} v$ to $d$. In particular, we want to know when $|\delta| \leq \frac{1}{q-p-1}<1$. The first inequality is obtained by modifying Lemma 4.4 in [13] for $\gamma v+\delta A v$. The last inequality holds if and only if $p<q-2$. Note that $q-p-1>0$ if $p<q-2$. When $p \geq q-2$, we have three cases.

Case 1. $p=q-2$ and $2 p=2(q-2) \leq q+3$ yields $q \leq 7$. Thus this case occurs when $(p, q)=(3,5),(5,7)$ since $p \neq 1$ and $q$ is odd.

Case 2. $p=q-1$ and $2 p=2(q-1) \leq q+3$ forces $q \leq 5$. In this case, we get $(p, q)=(2,3),(4,5)$.

Case 3. $p=q$ and $2 p=2 q \leq q+3$ gives $q \leq 3$. Then $(p, q)=(3,3)$ since $q$ is odd. 
In all these cases, $\Delta=p^{2}-4 q<0$. Then we can use the inequality $|\delta|<$ $\frac{2}{(\sqrt{q}-1)\left(\sqrt{4 q-p^{2}}\right)}$, which is derived by modifying (4.10) in Lemma 4.7 of [13] for $\gamma v+\delta A v=\sum_{k=2}^{\infty} A^{-(k-1)} \epsilon_{k}^{\prime} v$. Using this inequality, in cases 1 and 2 , we can see that $|\delta|<1$ by direct computation. When $p=q=3$, we get $|\delta|<2$.

Let $d=\gamma^{\prime} v+\delta^{\prime} A v \in T-T$. We have two possibilities for the case $2 p \leq q+3$ and $p \neq 1$.

(D-I) $2 p \leq q+2$ and $p \neq 1$ : Then the proof of Theorem 4.1 in 13 shows that possible nonzero integer vectors for $\sum_{k=1}^{\infty} A^{-k} b_{k} v$ are $\pm v, \pm(p v+A v), \pm((p-1) v+$ $A v)$. This case automatically excludes the pair $(p, q) \neq(3,3)$. Therefore, it follows that $\delta^{\prime}$, the second coordinate of $d$, can only be $0, \pm 1$. We use a technique to find candidates for neighbors of $T$. If $\gamma^{\prime} v+\delta^{\prime} A v=\epsilon_{1}^{\prime} v+b_{1} A^{-1} v+\sum_{k=2}^{\infty} A^{-k}\left(b_{k} v+\right.$ $\left.\epsilon_{k}^{\prime} A v\right) \in T-T$, where $\left|b_{k}\right| \leq q-1$, and $\epsilon_{k}^{\prime}=0, \pm 1$, then multiplying by $A$, we get $\gamma^{\prime \prime} v+\delta^{\prime \prime} A v \in T-T$, where $b_{1} v+\epsilon_{1}^{\prime} A v \in D-D, \gamma^{\prime \prime}=-\left(q \delta^{\prime}+b_{1}\right)$ and $\delta^{\prime \prime}=\gamma^{\prime}-p \delta^{\prime}-\epsilon_{1}^{\prime}, \epsilon_{1}^{\prime}=0, \pm 1$. This, in turn, implies that $\gamma^{\prime \prime \prime} v+\delta^{\prime \prime \prime} A v \in T-T$, where $\gamma^{\prime \prime \prime}=-\left(q \delta^{\prime \prime}+b_{2}\right)$ and $\delta^{\prime \prime \prime}=\gamma^{\prime \prime}-p \delta^{\prime \prime}-\epsilon_{2}^{\prime}, \epsilon_{2}^{\prime}=0, \pm 1$, etc.

We already know that $\delta^{\prime}=0$ and $\gamma^{\prime}= \pm 1$ give neighbors of $T$ by $(\mathrm{A})$ and $(\mathrm{B})$ above. If $\delta^{\prime}=0$ and $\gamma^{\prime} \neq 0, \pm 1$, then the only possibility is $\gamma^{\prime}= \pm 2, \epsilon_{1}^{\prime}= \pm 1$ and $\delta^{\prime \prime}= \pm 1$ since we must have $\left|\delta^{\prime \prime}\right|=\left|\gamma^{\prime}-\epsilon_{1}^{\prime}\right| \leq 1$ by our findings for a neighbor above.

We show that $\pm 2 v+T$ cannot be neighbors of $T$. Assume that $\pm 2 v \in T-T$ and get a contradiction. For the case $\delta^{\prime}=0, \gamma^{\prime}=2, \epsilon_{1}^{\prime}=1$ and $\delta^{\prime \prime}=1$, we have $\gamma^{\prime \prime}=$ $-\left(\delta^{\prime} q+b_{1}\right)=-b_{1}$. We will show below that when $\delta^{\prime \prime}=1$ the only possibilities for $\gamma^{\prime \prime}$ are $p-2, p-1, p, p+1, p+2$. Therefore, $b_{1}=-(p-2),-(p-1),-p,-(p+1),-(p+2)$. Note that $p \geq 2$ forces $b_{1} \leq 0$. Then $2 v=A^{-1}\left[\begin{array}{c}b_{1} \\ 1\end{array}\right]+\sum_{k=2}^{\infty}\left(b_{k} v+\epsilon_{k}^{\prime} A v\right) \in$ $T-T$. Since the sign of $b_{1}$ is nonpositive, $\left[\begin{array}{c}b_{1} \\ 1\end{array}\right]$ cannot be in $D-D$, which is a contradiction. Thus the assumption that $\pm 2 v \in T-T$ is wrong.

When $\delta^{\prime}=1,\left|\delta^{\prime \prime}\right|=\left|\gamma^{\prime}-p-\epsilon_{1}^{\prime}\right| \leq 1$ implies that $\gamma^{\prime}=p-2, p-1, p, p+1, p+2$. When $\delta^{\prime}=-1,\left|\delta^{\prime \prime}\right|=\left|\gamma^{\prime}+p-\epsilon_{1}^{\prime}\right| \leq 1$ implies that $\gamma^{\prime}=-(p-2),-(p-1),-p,-(p+$ $1),-(p+2)$.

We next argue that $\gamma^{\prime}=p$ and $\delta^{\prime}=1$ don't yield a neighbor. Suppose that $p v+A v \in T-T$. Then $\delta^{\prime \prime}=\gamma^{\prime}-p-\epsilon_{1}^{\prime}=p-p-\epsilon_{1}^{\prime}=-\epsilon_{1}^{\prime}$ and $\gamma^{\prime \prime}=-\left(q+b_{1}\right)<0$ forces $\epsilon_{1}^{\prime}=0,1$ because $\epsilon_{1}^{\prime}=-1$ would give $\delta^{\prime \prime}>0$ and $\gamma^{\prime \prime}=-\left(q+b_{1}\right)<0$ is not possible by the previous paragraph. If $\epsilon_{1}^{\prime}=0$, then $\left|\gamma^{\prime \prime}\right|=\left|-\left(q+b_{1}\right)\right| \leq 1$ yields $b_{1}=-(q-1)$. Hence $p v+A v=A^{-1}\left[\begin{array}{c}-(q-1) \\ 0\end{array}\right]+\sum_{k=2}^{\infty} A^{-k}\left(b_{k} v+\epsilon_{k}^{\prime} A v\right) \in T-T$, where $\left|b_{k}\right| \leq q-1,\left|\epsilon_{k}^{\prime}\right| \leq 1$. This is a contradiction since $\left[\begin{array}{c}-(q-1) \\ 0\end{array}\right] \notin D-D$. If $\epsilon_{1}^{\prime}=1$, then $\delta^{\prime \prime}=-1$, and $\gamma^{\prime \prime}=-\left(q+b_{1}\right)<0$ yields $q+b_{1}=p-2, p-1, p$ or $\gamma^{\prime \prime}=-p,-(p-1),-(p-2)$ because we will see below that $\delta^{\prime \prime}=-1$ and $\gamma^{\prime \prime}=-(p+1),-(p+2)$ don't yield neighbors. Thus $b_{1}=p-q, p-q-1, p-q-2$. But $p \leq q$, so $b_{1}=p-q, p-q-1, p-q-2 \leq 0$. Hence $p v+A v=A^{-1}\left[\begin{array}{c}b_{1} \\ 1\end{array}\right]+$ $\sum_{k=2}^{\infty} A^{-k}\left(b_{k} v+\epsilon_{k}^{\prime} A v\right) \in T-T$. This is a contradiction since $b_{1} \leq 0$ implies $\left[\begin{array}{c}b_{1} \\ 1\end{array}\right] \notin D-D$.

We then show that $\gamma^{\prime}=p+2$ and $\delta^{\prime}=1$ don't yield a neighbor. Suppose that $\gamma^{\prime}=p+2$ and $\delta^{\prime}=1$ gives a neighbor. Then $\delta^{\prime \prime}=\gamma^{\prime}-p \delta^{\prime}-\epsilon_{1}^{\prime}=p+2-p-\epsilon_{1}^{\prime}=$ $2-\epsilon_{1}^{\prime} \geq 1$ implies that $\delta^{\prime \prime \prime}=\gamma^{\prime \prime}-p \delta^{\prime \prime}-\epsilon_{2}^{\prime} \leq-\left(q \delta^{\prime}+b_{1}\right)-p-\epsilon_{2}^{\prime} \leq-q+(q-1)-p-\epsilon_{2}^{\prime}=$ $-p-1-\epsilon_{2}^{\prime} \leq-2$ since $p \geq 2$ and $\left|\epsilon_{2}^{\prime}\right| \leq 1$. This contradicts with $\left|\delta^{\prime \prime \prime}\right| \leq 1$. 
So far, we have shown that $2 v+T, p v+A v+T$ and $(p+2) v+A v+T$ are not neighbors of $T$. We finally suppose that $(p+1) v+A v+T$ is a neighbor of $T$, i.e, $(p+1) v+A v=\epsilon_{1}^{\prime} v+b_{1} v+\sum_{k=2}^{\infty} A^{-k}\left(b_{k} v+\epsilon_{k}^{\prime} A v\right) \in T-T$, where $\left|b_{k}\right| \leq q-1$, $\left|\epsilon_{k}^{\prime}\right| \leq 1$. Then $\delta^{\prime \prime}=\gamma^{\prime}-p \delta^{\prime}-\epsilon_{1}^{\prime}=p+1-p-\epsilon_{1}^{\prime}=1-\epsilon_{1}^{\prime} \geq 1$ for $\epsilon_{1}^{\prime} \neq 1$, and we proceed as in the previous paragraph to get the contradiction $\delta^{\prime \prime \prime} \leq-2$. If $\epsilon_{1}^{\prime}=1$, then $\delta^{\prime \prime}=0$ and we must have $\left|\gamma^{\prime \prime}\right|=\left|-\left(q \delta^{\prime}+b_{1}\right)\right|=\left|-\left(q+b_{1}\right)\right| \leq 1$ for a potential neighbor. This leads to $b_{1}=-(q-1)$. Then $(p+1) v+A v=v-(q-1) A^{-1} v+$ $\sum_{k=2}^{\infty} A^{-k}\left(b_{k} v+\epsilon_{k}^{\prime} A v\right)=A^{-1}\left[\begin{array}{c}-(q-1) \\ 1\end{array}\right]+\sum_{k=2}^{\infty} A^{-k}\left(b_{k} v+\epsilon_{k}^{\prime} A v\right) \in T-T$. This is impossible since $\left[\begin{array}{c}-(q-1) \\ 1\end{array}\right] \notin D-D$.

Thus $(p+1) v+A v+T$ is not a neighbor of $T$, and so $\pm v+T, \pm((p-1) v+A v)+T$ and $\pm((p-2) v+A v)+T$ form the neighbors of $T$ in this case. Theorem 2.1 and Theorem 2.3 imply that $T$ is disk-like.

(D-II) $2 p=q+3$ : We must necessarily have $p \geq 3$ since we assume that $q \geq 3$. This case includes the pairs $(p, q)=(3,3),(4,5),(5,7)$ above. Let $\Delta=p^{2}-4 q=$ $p^{2}-4(2 p-3)=p^{2}-8 p+12$. We have three cases:

(1) $\Delta>0$ : Then we obtain $p>6, q>9$ and $\left|\delta^{\prime}\right| \leq \frac{q}{q-p+1}=\frac{q}{q-\frac{q+3}{2}+1}=\frac{2 q}{q-1}<3$. The inequality for $\left|\delta^{\prime}\right|$ is a slightly changed version of the inequality in case (ii) of the proof of Proposition 4.5 in $\left[13\right.$ for $\gamma^{\prime} v+\delta^{\prime} A v \in T-T$.

(2) $\Delta=0$ : Since $p \geq 3$ and $2 p=q+3$, we have $p=6, q=9$ and $x^{2}+p x+q=$ $(x+3)^{2}$. But we are assuming in this section that $x^{2}+p x+q$ is irreducible. So $\Delta=0$ doesn't occur in our consideration here.

(3) $\Delta<0$ : In this case, $3 \leq p \leq 5$ and so $(p, q)=(3,3),(4,5),(5,7)$. Let $A^{-k} v:=\alpha_{k} v+\beta_{k} A v, k=1,2, \ldots$. We use the inequality $\left|\delta^{\prime}\right| \leq q\left(\frac{p}{q^{2}}+\frac{2 . q^{-1}}{(\sqrt{q}-1) \sqrt{-\Delta}}\right)$, which is again derived by modifying the first inequality of (4.10) with $j=3$ in Lemma 4.7 in 13 for $\gamma^{\prime} v+\delta^{\prime} A v$. This modification is obtained by taking into account the inequality $\beta_{1}=-q^{-1}<0$. Then we get $\left|\delta^{\prime}\right|<2$ for $p=4,5$. For $q=p=3, \beta_{1}, \beta_{3}<0$ and we need the sharper estimate $\left|\delta^{\prime}\right| \leq q\left(\frac{p}{q^{2}}+\frac{2 \cdot q^{-1}}{(\sqrt{q}-1) \sqrt{-\Delta}}-\beta_{3}\right)$ to conclude that $\left|\delta^{\prime}\right|<2$ again.

Now consider the case $\Delta>0$. In this case, we recall that $p>6, q>9$ and $\left|\delta^{\prime}\right|<3$. Furthermore, it is easy to show that $\beta_{k}=\frac{y_{2}^{k}-y_{1}^{k}}{\sqrt{\Delta}}$, where $y_{1}=\frac{-p+\sqrt{\Delta}}{2 q}, y_{2}=$ $\frac{-p+\sqrt{\Delta}}{2 q}<0$ are the roots of $q x^{2}+p x+1$. Then we study the case $\left|\delta^{\prime}\right|=2$. For this, it suffices to consider $\delta^{\prime}=2$. Since $\left|\delta^{\prime}\right| \leq \frac{q}{q-p+1}=\frac{2 q}{q-1}<3$ by (1), we may get $\delta^{\prime}=2$ only if we replace $q$ in the numerator by $q-1$. (That can be seen, just as in the case of $2 q$-adic representations, by considering the explicit expression of $\left.\beta_{k}\right)$. This happens when $\left(\sum_{k=1}^{\infty} A^{-2 k}-\sum_{k=1}^{\infty} A^{-(2 k-1)}\right)(q-1) v=\left[\begin{array}{l}\dot{2} \\ 2\end{array}\right]$. But such a series cannot be in $T-T$ because the first digit $-\left[\begin{array}{c}q-1 \\ 0\end{array}\right]$ is not in $D-D$. Thus $\gamma^{\prime} v+2 A v \in T-T$ is not possible. Thus we must have $\left|\delta^{\prime}\right|<2$. This reduces our proof to the case (D-I). Hence we conclude in this case that $\pm v+T, \pm((p-1) v+A v)+T$ and $\pm((p-2) v+A v)+T$ form the neighbors of $T$ as shown in (D-I).

Note that the characteristic polynomial in Proposition 3.6 satisfies $x^{2}-p x+q=$ $(-1)^{2}\left((-x)^{2}+p(-x)+q\right)$ so that Lemma 2.7 holds. But $D$ below doesn't satisfy the hypothesis of Proposition 2.5. That is why we cannot use Proposition 2.5 and 
Lemma 2.7 together with Proposition 3.5 and we need a separate proof for the following proposition.

Proposition 3.6. For any expanding matrix $A \in M_{2}(\mathbb{Z})$ with characteristic polynomial $f(x)=x^{2}-p x+q$, where $q$ is odd and $p, q>0$, there exists a noncollinear digit set $D$ so that $T(A, D)$ is a connected tile and is disk-like if and only if $2 p \leq q+3$ and $p \neq 1$. (See Figure 3.)

Proof. Let $A$ be the companion matrix of $x^{2}-p x+q$ and let

$$
D=\left\{\left[\begin{array}{l}
0 \\
0
\end{array}\right],\left[\begin{array}{c}
k \\
-1
\end{array}\right]: 1 \leq k \leq q-1\right\},
$$

which is slightly different from the one in the previous proof. We proceed as before.

(A) For $p=1$, we see from Table 2 that $T \pm\left[\begin{array}{l}1 \\ 0\end{array}\right], T \pm\left[\begin{array}{c}p-1 \\ -1\end{array}\right], T \pm\left[\begin{array}{c}p-2 \\ 1\end{array}\right]$, $T \pm\left[\begin{array}{c}p-2 \\ -1\end{array}\right]$ are neighbors of $T$.

Next we will show that $T \pm\left[\begin{array}{l}1 \\ 0\end{array}\right], T \pm\left[\begin{array}{c}p-1 \\ -1\end{array}\right], T \pm\left[\begin{array}{c}p-2 \\ -1\end{array}\right]$ are neighbors of $T$ for $p=2$. Compared with Proposition 3.5, it is somehow harder to find the neighbors here.

Assume that $p=2$. We use the digit set $D^{\prime}=\left[\begin{array}{l}1 \\ 0\end{array}\right]+D$ above. Then $A^{-1} d_{1}-$ $A^{-2} d_{q}=d_{1}$ implies that $S_{1}=A^{-1} d_{1}-A^{-2} d_{1}+A^{-3} d_{q}=0$.

$T \pm\left[\begin{array}{c}-1 \\ 1\end{array}\right]$ are neighbors of $T: \quad-A^{-1} d_{q}=-\left[\begin{array}{c}p-1 \\ -1\end{array}\right]=\left[\begin{array}{c}-1 \\ 1\end{array}\right]$ implies

$\left[\begin{array}{c}-1 \\ 1\end{array}\right]=S_{1}-A^{-1} d_{q}+\sum_{k=0}^{\infty}\left(A^{-(6 k+1)}+A^{-(6 k+6)}-A^{-(6 k+3)}-A^{-(6 k+4)}\right) S_{1} \in T-T$.

$T \pm\left[\begin{array}{c}-1 \\ 0\end{array}\right]$ are neighbors of $T$ :

$$
\begin{aligned}
{\left[\begin{array}{c}
-1 \\
0
\end{array}\right] } & =-A^{-1} d_{1}+A^{-2} d_{q}+S_{1} \\
& +\sum_{k=0}^{\infty}\left(A^{-(6 k+5)}+A^{-(6 k+6)}-A^{-(6 k+2)}-A^{-(6 k+3)}\right) S_{1} \in T-T .
\end{aligned}
$$

$T \pm\left[\begin{array}{l}0 \\ 1\end{array}\right]$ are neighbors of $T:$

$$
\begin{aligned}
-\left[\begin{array}{c}
p-2 \\
-1
\end{array}\right] & =\left[\begin{array}{l}
0 \\
1
\end{array}\right]=A^{-1} d_{1}-A^{-2} d_{q}-A^{-1} d_{q} \\
& +\sum_{k=0}^{\infty}\left(A^{-(6 k+1)}+A^{-(6 k+2)}-A^{-(6 k+4)}-A^{-(6 k+5)}\right) S_{1} \in T-T .
\end{aligned}
$$

By Theorem 2.1, $T$ is connected in this case.

(B) We now assume that $p>2$. Table 2 shows that $T \pm\left[\begin{array}{c}p-2 \\ -1\end{array}\right], T \pm\left[\begin{array}{c}p-1 \\ -1\end{array}\right]$, $T \pm\left[\begin{array}{l}1 \\ 0\end{array}\right]$, are neighbors of $T$. Theorem 2.1] implies that $T$ is connected again.

(C) When $2 p>q+3$ or $p=1, T$ is not disk-like: We now assume that $2 p>q+3$ so that $2(p-1) \geq q+2$. Then by the last line of Table 2, $T+d_{2 p-q-2}$ is also a neighbor of $T$. Therefore, $T(A, D)$ is not disk-like by Theorem 2.2 and (B) above. 
TABLE 2

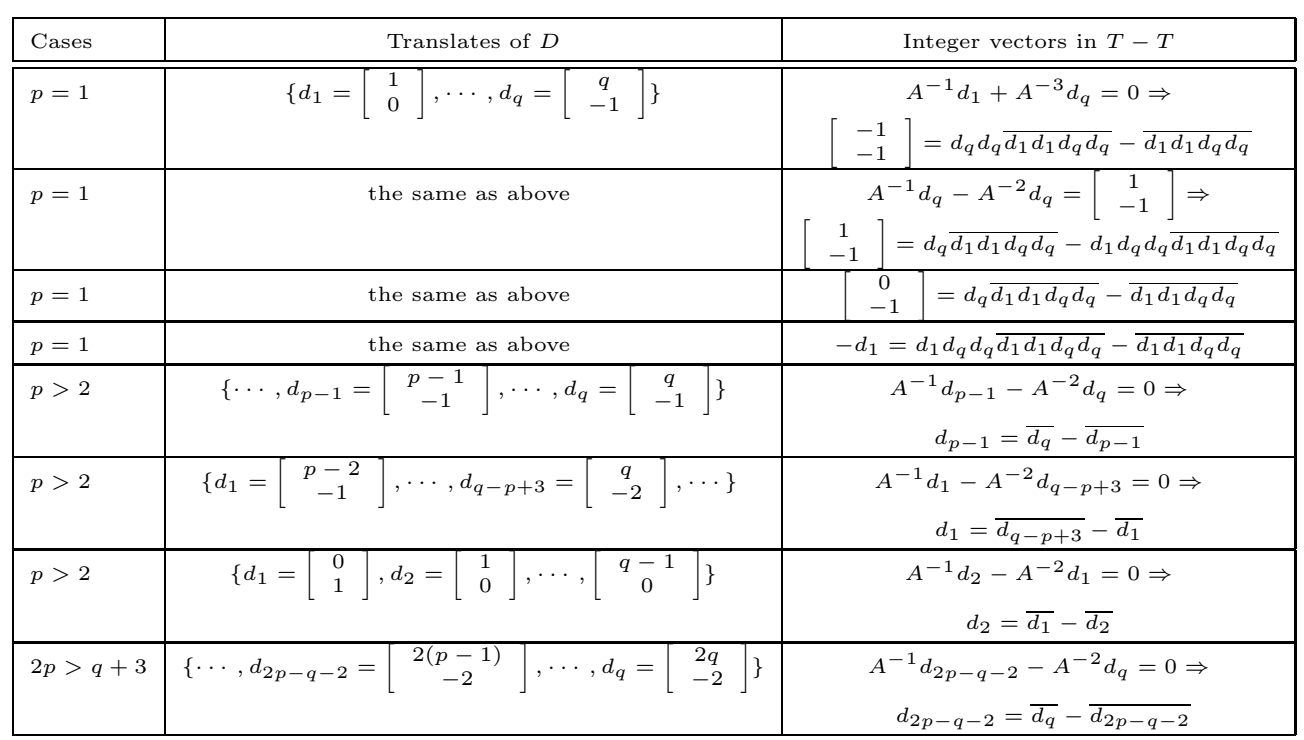

The neighbors of $T$ in (A) for $p=1$ are in the form of Theorem 2.2(ii). For $p=1$, we consider the translated digit set $D^{\prime}=\left[\begin{array}{l}1 \\ 1\end{array}\right]+D=\left\{d_{1}=\left[\begin{array}{l}1 \\ 1\end{array}\right], d_{2}=\right.$ $\left.\left[\begin{array}{l}2 \\ 0\end{array}\right],\left[\begin{array}{l}3 \\ 0\end{array}\right], \cdots, d_{q}=\left[\begin{array}{l}q \\ 0\end{array}\right]\right\}$ and we will show that $T \pm\left[\begin{array}{l}2 \\ 0\end{array}\right]$ is the fifth pair of neighbors of $T$ so that $T$ is not disk-like by Theorem 2.2 and $(\mathrm{A})$ in this proof. For this, we note that $A^{-1} d_{1}-A^{-2} d_{q}=d_{2}$ gives

$$
S_{2}:=A^{-1} d_{2}-A^{-2} d_{1}+A^{-3} d_{q}=0 .
$$

Then

$$
\left[\begin{array}{l}
2 \\
0
\end{array}\right]=A^{-1} d_{1}-A^{-2} d_{q}-S_{2}+\sum_{k=0}^{\infty}\left(A^{-(6 k+2)}+A^{-(6 k+3)}-A^{-(6 k+5)}-A^{-(6 k+6)}\right) S_{2} .
$$

This takes care of the case $p=1$.

(D) When $2 p \leq q+3$ and $p \neq 1$, there are no other neighbors: As in the proof of Proposition 3.5, with obvious modifications, we can show that $T \pm\left[\begin{array}{l}1 \\ 0\end{array}\right]$, $T \pm\left[\begin{array}{c}p-1 \\ -1\end{array}\right], T \pm\left[\begin{array}{c}p-2 \\ -1\end{array}\right]$ are the only neighbors of $T$ and $T$ is disk-like when $2 p \leq q+3$ and $p \neq 1$.

Proposition 3.7. For any expanding matrix $A \in M_{2}(\mathbb{Z})$ with characteristic polynomial $f(x)=x^{2}+p x+q$, where $q>0$ is even and $p \neq 0$, there exists a noncollinear digit set $D$ so that $T(A, D)$ is a connected tile and is disk-like if and only if $2|p| \leq|q+2|$. (See Figure 3 ,)

Proof. Let $A$ be the companion matrix of $x^{2}+p x+q$ and

$$
D=\left\{\left[\begin{array}{c}
2 k \\
0
\end{array}\right],\left[\begin{array}{c}
2 k \\
-1
\end{array}\right]: 0 \leq k \leq \frac{q-2}{2}\right\} .
$$



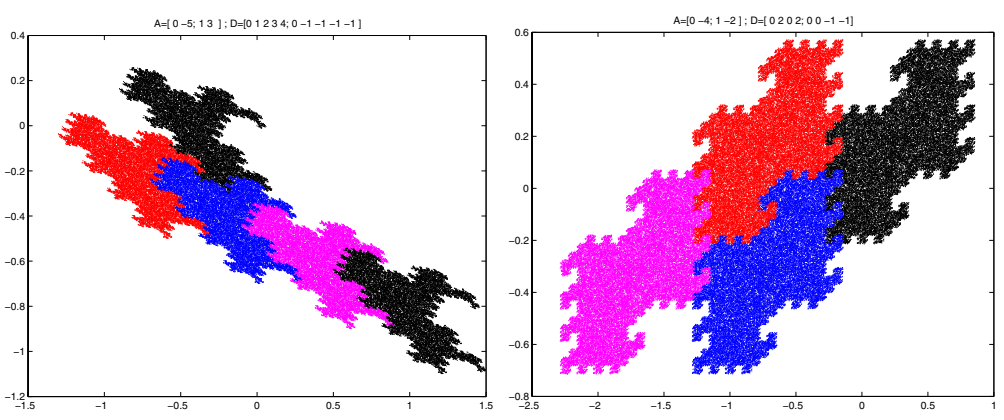

Figure 3. A disk-like tile of Proposition 3.6 (left) and a disk-like tile of Proposition 3.7

Note that $-A$ has characteristic polynomial $x^{2}-p x+q$ by Lemma 2.7. Also $D=$ $\left[\begin{array}{c}q-2 \\ -1\end{array}\right]-D$. Then $T(-A, D)=T(A, D)-\left(A-A^{-1}\right)^{-1}\left[\begin{array}{c}q-2 \\ -1\end{array}\right]$ by Proposition 2.5. Thus we only need to consider $A$ with $x^{2}+p x+q, p>0$. Note that $D$ is not a primitive digit set. Hence we transform it to a primitive complete digit set so that Theorem 3.2 applies. For this purpose, we let $B^{-1}=\left[\begin{array}{cc}\frac{1}{2} & 0 \\ 0 & 1\end{array}\right]$. Then

$$
B^{-1} A B=\left[\begin{array}{cc}
0 & \frac{-q}{2} \\
2 & -p
\end{array}\right],
$$

and $B^{-1} D=\left\{\left[\begin{array}{l}0 \\ 0\end{array}\right],\left[\begin{array}{l}1 \\ 0\end{array}\right], \cdots,\left[\begin{array}{c}\frac{q-2}{2} \\ 0\end{array}\right],\left[\begin{array}{c}0 \\ -1\end{array}\right],\left[\begin{array}{c}1 \\ -1\end{array}\right], \cdots,\left[\begin{array}{c}\frac{q-2}{2} \\ -1\end{array}\right]\right\}$. Thus we can assume that $A=\left[\begin{array}{cc}0 & \frac{-q}{2} \\ 2 & -p\end{array}\right]$, and

$$
D=\left\{\left[\begin{array}{l}
0 \\
0
\end{array}\right],\left[\begin{array}{l}
1 \\
0
\end{array}\right],\left[\begin{array}{l}
2 \\
0
\end{array}\right], \cdots,\left[\frac{q-2}{2}\right],\left[\begin{array}{c}
0 \\
-1
\end{array}\right],\left[\begin{array}{c}
1 \\
-1
\end{array}\right],\left[\begin{array}{c}
2 \\
-1
\end{array}\right], \cdots,\left[\frac{q-2}{-1}\right]\right\}
$$

so that $D$ is a primitive complete digit set and $\mu(T)=1$ by Theorem 3.2 ,

(A) We have two cases.

Case 1. $p$ is odd: We consider the translated digit set

$$
\begin{aligned}
D^{\prime} & =\left[\begin{array}{l}
1 \\
0
\end{array}\right]+D \\
& =\left\{d_{1}=\left[\begin{array}{l}
1 \\
0
\end{array}\right], \cdots,\left[\begin{array}{c}
\frac{q}{2} \\
0
\end{array}\right],\left[\begin{array}{c}
1 \\
-1
\end{array}\right], \cdots, d_{\frac{q+p+1}{2}}=\left[\begin{array}{c}
\frac{p+1}{2} \\
-1
\end{array}\right], \cdots, d_{q}=\left[\begin{array}{c}
\frac{q}{2} \\
-1
\end{array}\right]\right\} .
\end{aligned}
$$

We will show that $T \pm\left[\begin{array}{l}1 \\ 0\end{array}\right], T \pm\left[\begin{array}{c}\frac{p-1}{2} \\ 1\end{array}\right], T \pm\left[\begin{array}{c}\frac{p+1}{2} \\ 1\end{array}\right]$ are neighbors of $T$ so that $T$ is connected by Theorem 2.1. Note that $A^{-1} d_{\frac{q+p+1}{2}}+A^{-2} d_{q}=-d_{1}$ implies that

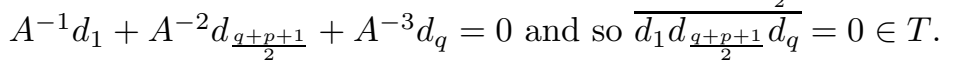

$T \pm\left[\begin{array}{l}1 \\ 0\end{array}\right]$ are neighbors of $T: \quad d_{\frac{q+p+1}{2}} d_{q} \overline{d_{1} d_{\frac{q+p+1}{2}} d_{q}}-\overline{d_{1} d_{\frac{q+p+1}{2}} d_{q}}=-d_{1} \in T-T$. $T \pm\left[\begin{array}{c}\frac{p+1}{2} \\ 1\end{array}\right]$ are neighbors of $T: \quad d_{q} \overline{d_{1} d_{\frac{q+p+1}{2}} d_{q}}-\overline{d_{1} d_{\frac{q+p+1}{2}} d_{q}}=-\left[\begin{array}{c}\frac{p+1}{2} \\ 1\end{array}\right] \in T-T$. $T \pm\left[\begin{array}{c}\frac{p-1}{2} \\ 1\end{array}\right]$ are neighbors of $T: \quad$ Next, we consider the digit set $D^{\prime}=\left[\begin{array}{l}1 \\ 1\end{array}\right]+$ $D=\left\{d_{1}=\left[\begin{array}{l}1 \\ 1\end{array}\right], \cdots, d_{\frac{q}{2}}=\left[\begin{array}{c}\frac{q}{2} \\ 1\end{array}\right], \cdots, d_{q}=\left[\begin{array}{c}\frac{q}{2} \\ 0\end{array}\right]\right\}$. Suppose that $p>1$. Then $d_{\frac{p-1}{2}}=\left[\begin{array}{c}\frac{p-1}{2} \\ 1\end{array}\right] \in D^{\prime}$. Now $A^{-1} d_{\frac{p-1}{2}}+A^{-2} d_{\frac{q}{2}}=0$ implies that $\overline{d_{\frac{p-1}{2}} d_{\frac{q}{2}}}=0 \in T$.

Hence $-d_{\frac{p-1}{2}}=d_{\frac{q}{2}} \overline{d_{\frac{p-1}{2}} d_{\frac{q}{2}}}-\overline{d_{\frac{p-1}{2}} d_{\frac{q}{2}}} \in T-T$. 
We next assume that $p=1$. Then $A^{-1} d_{q}+A^{-2} d_{\frac{q}{2}}=-d_{1}$ implies $A^{-1} d_{1}+$ $A^{-2} d_{q}+A^{-3} d_{\frac{q}{2}}=0$. Then $\overline{d_{1} d_{q} d_{\frac{q}{2}}}=0 \in T$, and hence $-\left[\begin{array}{c}\frac{p-1}{2} \\ 1\end{array}\right]=-\left[\begin{array}{l}0 \\ 1\end{array}\right]=$ $d_{\frac{q}{2}} \overline{d_{1} d_{q} d_{\frac{q}{2}}}-\overline{d_{1} d_{q} d_{\frac{q}{2}}} \in T-T$.

We now assume that $2|p| \leq|q+2|$ and determine candidates for possible neighbors of $T$. Considering the translated digit set

$D+\left[\begin{array}{l}0 \\ 1\end{array}\right]=\left\{\left[\begin{array}{l}0 \\ 0\end{array}\right],\left[\begin{array}{l}1 \\ 0\end{array}\right],\left[\begin{array}{l}2 \\ 0\end{array}\right], \cdots,\left[\frac{q-2}{2}\right],\left[\begin{array}{l}0 \\ 1\end{array}\right],\left[\begin{array}{l}1 \\ 1\end{array}\right],\left[\begin{array}{l}2 \\ 1\end{array}\right], \cdots,\left[\begin{array}{c}\frac{q-2}{2} \\ 1\end{array}\right]\right\}$,

we see that any integer vector $d \in T-T$ has the form $\frac{1}{2}\left(\epsilon v+\sum_{k=1}^{\infty} A^{-k} b_{k} v\right)$, where $v=\left[\begin{array}{l}1 \\ 0\end{array}\right], \epsilon=0, \pm 1$, and $\left|b_{k}\right| \in\{0,1,2, \ldots, q-1\}$. The proof of Theorem 4.1 in [13] shows that possible nonzero integer vectors for $\sum_{k=1}^{\infty} A^{-k} b_{k} v$ are $\pm v, \pm((p-1) v+$ $A v), \pm(p v+A v)$. Thus any nonzero integer vector $d \in T-T$ has one of the forms $\pm v, \pm \frac{1}{2}((p-1) v+A v), \pm \frac{1}{2}((p+1) v+A v)$ since $p$ is odd. Therefore, the above discussion shows that the neighbors of $T$ consist of $T \pm v, T \pm \frac{1}{2}((p-1) v+A v)$, $T \pm \frac{1}{2}((p+1) v+A v)$. We conclude that $T$ is disk-like by Theorem 2.1 and Theorem 2.3 .

Case 2. $p$ is even: WLOG, we can study the translated digit set

$D^{\prime}=\left[\begin{array}{l}1 \\ 0\end{array}\right]+D=\left\{d_{1}=\left[\begin{array}{l}1 \\ 0\end{array}\right], \cdots, d_{\frac{q}{2}}=\left[\begin{array}{c}\frac{q}{2} \\ 0\end{array}\right], \cdots, d_{\frac{q+p}{2}}=\left[\begin{array}{c}\frac{p}{2} \\ -1\end{array}\right], \cdots,\left[\begin{array}{c}\frac{q}{2} \\ -1\end{array}\right]\right\}$.

We will show that $T \pm\left[\begin{array}{l}1 \\ 0\end{array}\right], T \pm\left[\begin{array}{c}\frac{p}{2} \\ 1\end{array}\right], T \pm\left[\begin{array}{c}\frac{p-2}{2} \\ 1\end{array}\right]$ are neighbors of $T$ so that $T$ is connected by Theorem 2.1, We first notice that $A^{-1} d_{\frac{q+p}{2}}+A^{-2} d_{\frac{q}{2}}=-d_{1}$ implies that $\overline{d_{1} d_{\frac{q+p}{2}} d_{\frac{q}{2}}}=0 \in T$.

$T \pm\left[\begin{array}{l}1 \\ 0\end{array}\right]$ are neighbors of $T: \quad d_{\frac{q+p}{2}} d_{\frac{q}{2}} \overline{d_{1} d_{\frac{q+p}{2}} d_{\frac{q}{2}}}-\overline{d_{1} d_{\frac{q+p}{2}} d_{\frac{q}{2}}}=-d_{1} \in T-T$.

$T \pm\left[\begin{array}{c}\frac{p}{2} \\ 1\end{array}\right]$ are neighbors of $T: \quad d_{\frac{q}{2}} \overline{d_{1} d_{\frac{q+p}{2}} d_{\frac{q}{2}}}-\overline{d_{1} d_{\frac{q+p}{2}} d_{\frac{q}{2}}}=-\left[\begin{array}{c}\frac{p}{2} \\ 1\end{array}\right] \in T-T$.

$T \pm\left[\begin{array}{c}\frac{p-2}{2} \\ 1\end{array}\right]$ are neighbors of $T$ : We may study the translated digit set

$$
\begin{aligned}
D^{\prime} & =\left[\begin{array}{l}
1 \\
2
\end{array}\right]+D \\
& =\left\{\left[\begin{array}{l}
1 \\
2
\end{array}\right], \cdots, d_{\frac{q}{2}}=\left[\begin{array}{c}
\frac{q}{2} \\
2
\end{array}\right], d_{\frac{q+2}{2}}=\left[\begin{array}{l}
1 \\
1
\end{array}\right], \cdots, d_{q}=\left[\begin{array}{c}
\frac{q}{2} \\
1
\end{array}\right]\right\} .
\end{aligned}
$$

We first suppose that $p>2$ so that $\frac{d_{\frac{q+p-2}{2}}}{2}=\left[\begin{array}{c}\frac{p-2}{2} \\ 1\end{array}\right] \in D^{\prime}$. Then $A^{-1} d_{\frac{q+p-2}{2}}+$ $A^{-2} d_{\frac{q}{2}}=0$ implies that $\overline{d_{\frac{q+p-2}{2}} d_{\frac{q}{2}}}=0 \in T$.

This gives $d_{\frac{q}{2}} \overline{d_{\frac{q+p-2}{2}} d_{\frac{q}{2}}}-\overline{d_{\frac{q+p-2}{2}} d_{\frac{q}{2}}}=-d_{\frac{q+p-2}{2}} \in T$.

We next assume that $p=2$. Then $A^{-1} d_{q}+A^{-2} d_{\frac{q}{2}}=-d_{\frac{q+2}{2}}$ implies $A^{-1} d_{\frac{q+2}{2}}+$ $A^{-2} d_{q}+A^{-3} d_{\frac{q}{2}}=0$. Then $\overline{d_{\frac{q+2}{2}} d_{q} d_{\frac{q}{2}}}=0 \in T$ and hence $-\left[\begin{array}{c}\frac{p-2}{2} \\ 1\end{array}\right]=\left[\begin{array}{c}0 \\ -1\end{array}\right]=$ $d_{\frac{q}{2}} \overline{d_{\frac{q+2}{2}} d_{q} d_{\frac{q}{2}}}-\overline{d_{\frac{q+2}{2}} d_{q} d_{\frac{q}{2}}} \in T-T$.

We now suppose that $2|p| \leq|q+2|$ and we determine candidates for possible neighbors of $T$. Considering the translated digit set

$D+\left[\begin{array}{l}0 \\ 1\end{array}\right]=\left\{\left[\begin{array}{l}0 \\ 0\end{array}\right],\left[\begin{array}{l}1 \\ 0\end{array}\right],\left[\begin{array}{l}2 \\ 0\end{array}\right], \cdots,\left[\frac{q-2}{2}\right],\left[\begin{array}{l}0 \\ 1\end{array}\right],\left[\begin{array}{l}1 \\ 1\end{array}\right],\left[\begin{array}{l}2 \\ 1\end{array}\right], \cdots,\left[\frac{q-2}{2}\right]\right\}$, we see that any nonzero integer vector $d \in T-T$ has the form $\frac{1}{2}\left(\epsilon v+\sum_{k=1}^{\infty} A^{-k} b_{k} v\right)$, where $v=\left[\begin{array}{l}1 \\ 0\end{array}\right], \epsilon=0, \pm 1$, and $\left|b_{k}\right| \in\{0,1,2, \ldots, q-1\}$. The proof of Theorem 4.1 
in 13 shows that possible nonzero integer vectors for $\sum_{k=1}^{\infty} A^{-k} b_{k} v$ are $\pm v, \pm((p-$ 1) $v+A v), \pm(p v+A v)$. Thus any nonzero integer vector $d \in T-T \subseteq \mathbb{Z}^{2}$ has one of the forms $\pm v, \pm \frac{1}{2}((p-2) v+A v), \pm \frac{1}{2}(p v+A v)$. Therefore, the above discussion shows that the neighbors of $T$ consist of $T \pm v, T \pm \frac{1}{2}((p-2) v+A v), T \pm \frac{1}{2}(p v+A v)$. We again conclude that $T$ is disk-like by Theorem 2.3 .

(B) We now assume that $2|p|>|q+2|$ and prove that $T(A, D)$ is not disk-like by giving another neighbor of $T$. We now consider the translated digit set

$$
\begin{aligned}
D^{\prime} & =\left[\begin{array}{c}
\frac{q}{2}+1 \\
2
\end{array}\right]+D \\
& =\left\{\left[\begin{array}{c}
\frac{q}{2}+1 \\
1
\end{array}\right],\left[\begin{array}{c}
\frac{q}{2}+2 \\
1
\end{array}\right], \cdots,\left[\begin{array}{c}
q-1 \\
1
\end{array}\right],\left[\begin{array}{c}
q \\
1
\end{array}\right],\left[\begin{array}{c}
\frac{q}{2}+1 \\
2
\end{array}\right], \cdots, d_{q}=\left[\begin{array}{c}
q \\
2
\end{array}\right]\right\}
\end{aligned}
$$

with $d_{p-1}=\left[\begin{array}{c}p-1 \\ 2\end{array}\right]$. Then it follows from the equalities $A^{-1} d_{p-1}+A^{-2} d_{q}=0$ and $A^{-1} d_{q}=-d_{p-1}$ that $d_{p-1}=\overline{d_{p-1} d_{q}}-d_{q} \overline{d_{p-1} d_{q}} \in T-T$. Therefore, $T+d_{p-1}$ is a neighbor of $T$. This neighbor is not in the form of Theorem 2.2 and so $T(A, D)$ is not disk-like.

Proposition 3.8. For any expanding matrix $A \in M_{2}(\mathbb{Z})$ with characteristic polynomial $f(x)=x^{2}+p x-q$, where $q$ is odd and $p, q>0$, there exists a noncollinear digit set $D$ so that $T(A, D)$ is a connected tile and is disk-like if and only if $2 p \leq q-3$.

Proof. Let $A=\left[\begin{array}{cc}0 & q \\ 1 & -p\end{array}\right]$, the companion matrix of $x^{2}+p x-q$. Note that $p \leq q-2$ by Lemma 2.6.

Let $D=\left\{\left[\begin{array}{l}0 \\ 0\end{array}\right],\left[\begin{array}{l}k \\ 1\end{array}\right]: 1 \leq k \leq q-1\right\}$.

(A) For $p+2=q, \sum_{k=1}^{\infty} A^{-k}=(A-I)^{-1}=\left[\begin{array}{cc}-1 & q \\ 1 & -p-1\end{array}\right]^{-1}=\left[\begin{array}{cc}p+1 & p+2 \\ 1 & 1\end{array}\right]$. Using the translated digit set in the first line of Table 3 , we have $\overline{d_{2} d_{1}}-\overline{d_{1}}=$ $\left[\begin{array}{c}p+2 \\ 1\end{array}\right] \in T-T$. Thus $T \pm\left[\begin{array}{c}p+2 \\ 1\end{array}\right]$ are neighbors of $T$. This together with the first three lines of Table 3 shows that $T \pm\left[\begin{array}{l}1 \\ 0\end{array}\right], T \pm\left[\begin{array}{c}p+1 \\ 1\end{array}\right], T \pm\left[\begin{array}{c}p+2 \\ 1\end{array}\right]$ are neighbors of $T$. This makes $T$ connected by Theorem 2.1 .

TABle 3

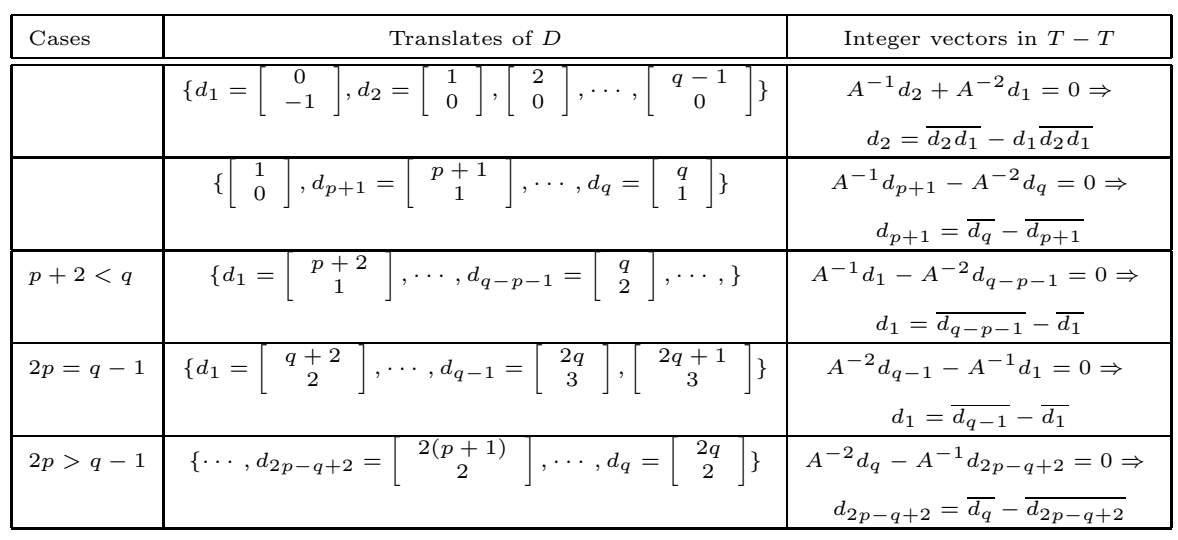

(B) When $2 p \geq q-1, T$ is not disk-like: If $2 p=q-1$, then from the fourth line of Table [3, we see that $T+\left[\begin{array}{c}q+2 \\ 2\end{array}\right]$ is another neighbor of $T$ and, in view of (A), 
$T$ is not disk-like by Theorem 2.2. For $2 p>q-1$, we have $2(p+1)>q+1$. In this case, the last line of Table 3 says that $\left[\begin{array}{c}2(p+1) \\ 2\end{array}\right]$ is also a neighbor of $T$ and so $T$ is not disk-like by Theorem 2.2 .

(C) We now assume that $2 p \leq q-3$. This case automatically excludes $q=3$ since $p \neq 0$, and hence we must have $q \geq 4$. We will show that there are no neighbors of $T$ other than $T \pm\left[\begin{array}{l}1 \\ 0\end{array}\right], T \pm\left[\begin{array}{c}p+1 \\ 1\end{array}\right], T \pm\left[\begin{array}{c}p+2 \\ 1\end{array}\right]$. In our case, we need more study of the elements of $T-T$ than that in 13 .

We note that any nonzero integer vector $d \in T-T$ has the form $d=\epsilon_{1}^{\prime} v+$ $b_{1} A^{-1} v+\sum_{k=2}^{\infty} A^{-k}\left(\epsilon_{k}^{\prime} v+A^{-k} b_{k} v\right)$, where $v=\left[\begin{array}{l}1 \\ 0\end{array}\right], \epsilon_{k}^{\prime}=0, \pm 1,\left|b_{k}\right| \leq q-1$.

Now we need to see the contribution of $\gamma v+\delta A v:=\sum_{k=2}^{\infty} A^{-k} \epsilon_{k}^{\prime} v$ to $d$. Let $A^{-k} v:=\alpha_{k} v+\beta_{k} A v, k=1,2, \ldots$. Similar to the proof of Lemma 4.4 in [13], we can use the recursion relation for $\alpha_{k}$ and $\beta_{k}$ in Proposition 4.2 of [13], and we can show that $|\gamma| \leq \frac{1+p}{q-p-1} \leq \frac{q-1}{q+1}<1$ using $2 p \leq q-3$, and also $|\delta| \leq \frac{1}{q-p-1} \leq 2 /(q+1)<1$ since $q \geq 4$. Then the proof of Theorem 4.1 in [13] shows that possible nonzero integer vectors for $\sum_{k=2}^{\infty} A^{-k} \epsilon_{k}^{\prime} v+\sum_{k=1}^{\infty} A^{-k} b_{k} v$ are $\pm v, \pm(p v+A v), \pm(p+1) v+A v$.

Suppose that $\epsilon_{1}^{\prime}=0$. Then $d=\sum_{k=2}^{\infty} A^{-k} \epsilon_{k}^{\prime} v+\sum_{k=1}^{\infty} A^{-k} b_{k} v$ has one of the forms $\pm v, \pm(p v+A v), \pm((p+1) v+A v)$ by the above observation. Suppose that $\epsilon_{1}^{\prime}= \pm 1$. Thus any nonzero vector $d \in T-T \subseteq \mathbb{Z}^{2}$ has one of the forms $\pm v, \pm 2 v, \pm((p-1) v+A v), \pm(p v+A v), \pm((p+1) v+A v)), \pm((p+2) v+A v)$.

If $\gamma^{\prime} v+\delta^{\prime} A v=\epsilon_{1}^{\prime} v+b_{1} A^{-1} v+\sum_{k=2}^{\infty} A^{-k}\left(b_{k} v+\epsilon_{k}^{\prime} A v\right) \in T-T$, where $\left|b_{k}\right| \leq$ $q-1$, and $\epsilon_{k}^{\prime}=0, \pm 1$, then multiplying by $A$, we get $\gamma^{\prime \prime} v+\delta^{\prime \prime} A v \in T-T$, where $b_{1} v+\epsilon_{1}^{\prime} A v \in D-D, \gamma^{\prime \prime}=-\left(-q \delta^{\prime}+b_{1}\right)$ and $\delta^{\prime \prime}=\gamma^{\prime}-p \delta^{\prime}-\epsilon_{1}^{\prime}, \epsilon_{1}^{\prime}=0, \pm 1$. Here, the thing that differs from (D-I) of the proof of Proposition [3.5] is the coefficient of $q \delta^{\prime}$ in $\gamma^{\prime \prime}=-\left(-q \delta^{\prime}+b_{1}\right)$, which is 1 . Then a case-by-case argument, which is shorter than (D-I), leads us to $\pm 2 v, \pm((p-1) v+A v), \pm(p v+A v) \notin T-T$ and we omit the details here.

Thus $\pm v+T, \pm((p+1) v+A v))+T, \pm((p+2) v+A v)+T$ form the neighbors of $T$, and $T$ is disk-like by Theorem 2.3 and (A) in this proof.

Proposition 3.9. For any expanding matrix $A \in M_{2}(\mathbb{Z})$ with characteristic polynomial $f(x)=x^{2}-p x-q$, where $q$ is odd and $p, q>0$, there exists a noncollinear digit set $D$ so that $T(A, D)$ is a connected tile and is disk-like if and only if $2 p \leq q-3$. (See Figure 4.)

Proof. Let $A$ be the companion matrix of $x^{2}-p x-q$. Again $p \leq q-2$ by Lemma 2.6. Let $D=\left\{\left[\begin{array}{l}0 \\ 0\end{array}\right],\left[\begin{array}{c}k \\ -1\end{array}\right]: 1 \leq k \leq q-1\right\}$.

(A) We assume that $p+2=q$. We now make use of the $\operatorname{digit}$ set $D^{\prime}=\left[\begin{array}{c}q-1 \\ -1\end{array}\right]+$ $D=\left\{d_{1}=\left[\begin{array}{c}q-1 \\ -1\end{array}\right], d_{2}=\left[\begin{array}{c}q \\ -2\end{array}\right],\left[\begin{array}{c}q+1 \\ -2\end{array}\right], \cdots,\left[\begin{array}{c}2(q-1) \\ -2\end{array}\right]\right\}$. Then $A^{-2} d_{2}=d_{1}$ implies that $A^{-3} d_{2}-A^{-1} d_{1}=0$. Since $A^{-1} d_{2}=\left[\begin{array}{c}-(p+2) \\ 1\end{array}\right]$, we obtain

$$
\begin{aligned}
{\left[\begin{array}{c}
-(p+2) \\
1
\end{array}\right] } & =A^{-1} d_{2}+\sum_{k=0}^{\infty} A^{-(4 k+1)}\left(A^{-1} d_{1}-A^{-3} d_{2}\right) \\
& +\sum_{k=0}^{\infty} A^{-(4 k)}\left(I+A^{-1}+A^{-2}\right)\left(A^{-3} d_{2}-A^{-1} d_{1}\right) \in T-T
\end{aligned}
$$


TABLE 4

\begin{tabular}{|c|c|c|c|c|c|c|c|}
\hline \multirow[t]{2}{*}{ Cases } & \multicolumn{6}{|c|}{ Translates of $D$} & Integer vectors in $T-T$ \\
\hline & $\left\{d_{1}=\right.$ & $\left.\begin{array}{l}0 \\
1\end{array}\right]$, & $=\left[\begin{array}{l}1 \\
= \\
0\end{array}\right.$ & \multicolumn{3}{|c|}{$\left.\left.\begin{array}{c|c}q-1 \\
0\end{array}\right]\right\}$} & $\begin{array}{c}A^{-1} d_{2}-A^{-2} d_{1}=0 \Rightarrow \\
d_{2}=\overline{d_{1}}-\overline{d_{2}}\end{array}$ \\
\hline & $\left\{\left[\begin{array}{l}1 \\
0\end{array}\right.\right.$ & $\cdots, d_{p+1}=$ & $=\left[\begin{array}{c}p+1 \\
-1\end{array}\right.$ & $\cdots, c$ & $l_{q}=$ & $\left.\begin{array}{c|c}q \\
-1\end{array}\right\}$ & $\begin{array}{c}A^{-1} d_{p+1}+A^{-2} d_{q}=0 \Rightarrow \\
d_{p+1}=\overline{d_{p+1} d_{q}}-d_{q} \overline{d_{p+1} d_{q}}\end{array}$ \\
\hline$p+2<q$ & $\left\{d_{1}=\right.$ & $\begin{array}{c}p+2 \\
-1\end{array}$ & $\cdots, d_{q-\gamma}$ & & $\begin{array}{c}q \\
-2\end{array}$ & $, \cdots\}$ & $\begin{array}{c}A^{-1} d_{1}+A^{-2} d_{q-p-1}=0 \Rightarrow \\
d_{1}=\overline{d_{1} d_{q-p-1}}-d_{q-p-1} \overline{d_{1} d_{q-p-1}}\end{array}$ \\
\hline $2 p=q-1$ & $\left\{d_{1}=\right.$ & $\left.\begin{array}{c}q+2 \\
-2\end{array}\right], \ldots$ &,$d_{q-1}=$ & $\begin{array}{l}2 q \\
-3\end{array}$ &, $2^{2}$ & $\left.\left.\begin{array}{c}2 q+1 \\
-3\end{array}\right\}\right\}$ & $\begin{array}{l}A^{-1} d_{1}+A^{-2} d_{q-1}=0 \Rightarrow \\
d_{1}=\overline{d_{1} d_{q-1}}-d_{q-1} \overline{d_{1} d_{q-1}}\end{array}$ \\
\hline $2 p>q-1$ & $\{\cdot, \cdots$ & $b-q+2=$ & $\begin{array}{c}2(p+1) \\
-2\end{array}$ & & $d_{q}=$ & $\left.=\left[\begin{array}{c}2 q \\
-2\end{array}\right]\right\}$ & $\begin{array}{c}A^{-1} d_{2 p-q+2}+A^{-2} d_{q}=0 \Rightarrow \\
d_{2 p-q+2}=\overline{d_{2 p-q+2} d_{q}}-d_{q} \overline{d_{2 p-q+2} d_{q}}\end{array}$ \\
\hline
\end{tabular}
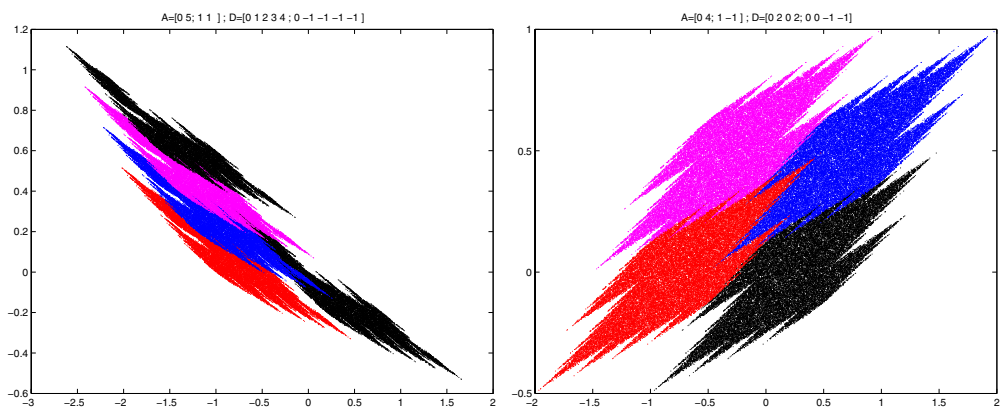

Figure 4. A disk-like tile of Proposition 3.9 (left) and a disk-like tile of Proposition 3.10 .

This together with the first three lines of Table 4 yields that $T \pm\left[\begin{array}{l}1 \\ 0\end{array}\right], T \pm$ $\left[\begin{array}{c}p+1 \\ -1\end{array}\right], T \pm\left[\begin{array}{c}p+2 \\ -1\end{array}\right]$ are neighbors of $T$ so that $T$ is connected by Theorem 2.1 .

(B) When $2 p \geq q-1, T$ is not disk-like: The last two lines of Table 4 say that $T$ has neighbors other than the ones in (A) above and they are not in the form of Theorem 2.2. Therefore, $T$ is not disk-like.

(C) Assume that $2 p \leq q-3$. As in (C) in the proof of Proposition 3.8, one can show that $\pm 2 v+T, \pm((p-1) v-A v)+T$ and $\pm(p v-A v)+T$ are not neighbors of $T$. Thus the neighbors of $T$ consist of $\pm v+T, \pm((p+1) v-A v)+T, \pm((p+2) v-A v)+T$ and $T$ is disk-like by Theorem 2.3 .

Remark. The proofs also give boundary points of the tiles. For instance, in the first line of Table 4, we have $d_{2}=\overline{d_{1}}-\overline{d_{2}}$ or $d_{2}+\overline{d_{2}}=\overline{d_{1}}$. This means that $\overline{d_{1}}$ is a boundary point.

Proposition 3.10. For any expanding matrix $A \in M_{2}(\mathbb{Z})$ with characteristic polynomial $x^{2}+p x-q$, where $q>0$ is even and $p \neq 0$, there exists a noncollinear digit set $D$ so that $T(A, D)$ is a connected tile and is disk-like if and only if $2|p| \leq|q+2|$. 
Proof. Instead of using the companion matrix of $x^{2}+p x-q$ and the digit set

$$
\left\{\left[\begin{array}{c}
2 k \\
0
\end{array}\right],\left[\begin{array}{c}
2 k \\
-1
\end{array}\right]: 0 \leq k \leq \frac{q-2}{2}\right\}
$$

we can assume that $A=\left[\begin{array}{cc}0 & \frac{q}{2} \\ 2 & -p\end{array}\right]$, and

$$
D=\left\{\left[\begin{array}{l}
0 \\
0
\end{array}\right],\left[\begin{array}{l}
1 \\
0
\end{array}\right],\left[\begin{array}{l}
2 \\
0
\end{array}\right], \cdots,\left[\begin{array}{c}
\frac{q-2}{2} \\
0
\end{array}\right],\left[\begin{array}{c}
0 \\
-1
\end{array}\right],\left[\begin{array}{c}
1 \\
-1
\end{array}\right],\left[\begin{array}{c}
2 \\
-1
\end{array}\right], \cdots,\left[\frac{q-2}{2}\right]\right\}
$$

as in the proof of Proposition 3.7. Note that $-A$ has characteristic polynomial $x^{2}-p x-q$ by Lemma 2.7. Also $D=\left[\frac{q-2}{2}\right]-D$. Then $T(-A, D)=T(A, D)-$ $\left(A-A^{-1}\right)^{-1}\left[\frac{\frac{q-2}{2}}{-1}\right]$ by Proposition 2.5 , and we only need to consider $A$ with $x^{2}+p x-q, p>0$. By Lemma 2.6, we have $p \leq q-2$.

(A) Compared with Proposition [3.7, it is a little harder to find the neighbors here. We have two cases.

Case 1. $p$ is odd: We will show that $T \pm\left[\begin{array}{l}1 \\ 0\end{array}\right], T \pm\left[\begin{array}{c}\frac{p-1}{2} \\ 1\end{array}\right], T \pm\left[\begin{array}{c}\frac{p+1}{2} \\ 1\end{array}\right]$ are neighbors of $T$ so that $T$ will be connected.

$T \pm\left[\begin{array}{l}1 \\ 0\end{array}\right]$ are neighbors of $T$ : Here we note that $\frac{p+3}{2} \leq \frac{q}{2}$ because $q$ is even, $p$ is odd and $p \leq q-2$. We consider the translated digit set $D^{\prime}=\left[\begin{array}{l}1 \\ 0\end{array}\right]+D$ with

$$
\begin{aligned}
D^{\prime}=\quad & \left\{d_{1}=\left[\begin{array}{l}
1 \\
0
\end{array}\right], \cdots, d_{\frac{q}{2}}=\left[\begin{array}{c}
\frac{q}{2} \\
0
\end{array}\right],\left[\begin{array}{c}
1 \\
-1
\end{array}\right], \cdots, d_{\frac{q+p-1}{2}}=\left[\begin{array}{c}
\frac{p-1}{2} \\
-1
\end{array}\right],\right. \\
& \left.d_{\frac{q+p+1}{2}}=\left[\begin{array}{c}
\frac{p+1}{2} \\
-1
\end{array}\right], d_{\frac{q+p+3}{2}}=\left[\begin{array}{c}
\frac{p+3}{2} \\
-1
\end{array}\right], \cdots, d_{q}=\left[\begin{array}{c}
\frac{q}{2} \\
-1
\end{array}\right]\right\} .
\end{aligned}
$$

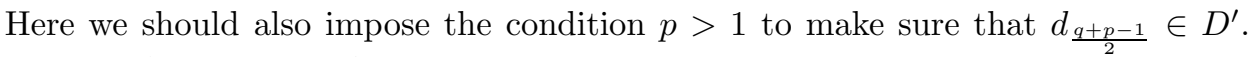
Since $A^{-1} d_{\frac{q+p-1}{2}}-A^{-2} d_{q}=-d_{1}$, we have

$$
S_{3}=\sum_{k=0}^{\infty} A^{-k}\left(A^{-1} d_{1}+A^{-2} d_{\frac{q+p-1}{2}}-A^{-3} d_{q}\right)=0 .
$$

Then,

$$
\begin{aligned}
-d_{1} & =A^{-1} d_{\frac{q+p-1}{2}}-A^{-2} d_{q}+S_{3}=\sum_{k=1}^{\infty} A^{-k} d_{\frac{q+p+1}{2}}-\sum_{k=2}^{\infty} A^{-k} d_{q} \\
& =A^{-1} d_{1}-A^{-1} d_{1}+\sum_{k=1}^{\infty} A^{-k} d_{\frac{q+p+1}{2}}-\sum_{k=2}^{\infty} A^{-k} d_{q} \\
& =d_{\frac{q+p+3}{2}} \overline{d_{\frac{q+p+1}{2}}}-d_{1} \overline{d_{q}} \in T-T .
\end{aligned}
$$

In the case $p=1$, notice that $d_{1}=A^{-1} d_{\frac{q}{2}}+A^{-2} d_{q}-A^{-1} d_{q}$ so that $A^{-2} d_{\frac{q}{2}}+$ $A^{-3} d_{q}-A^{-1} d_{1}-A^{-2} d_{q}=0$. Then

$$
d_{1}=d_{\frac{q}{2}} d_{q} \overline{d_{\frac{q}{2}} d_{q}}-d_{q} \overline{d_{1} d_{q}}
$$




$$
\begin{aligned}
& T \pm\left[\begin{array}{c}
\frac{p-1}{2} \\
1
\end{array}\right] \text { are neighbors of } T: \text { Let } D^{\prime}=\left[\begin{array}{l}
1 \\
0
\end{array}\right]+D \text { again. We have } A^{-1} d_{q}= \\
& \begin{aligned}
{\left[\frac{p-1}{1}\right]^{2} } & =d \text {. Then, } \\
d & =A^{-1} d_{q}-S_{3} \\
& =A^{-2} d_{1}-A^{-2} d_{1}+A^{-1} d_{q}+\sum_{k=3}^{\infty} A^{-k} d_{q}-A^{-1} d_{1}-\sum_{k=2}^{\infty} A^{-k} d_{\frac{q+p+1}{2}} \\
& =d_{q} d_{1} \overline{d_{q}}-d_{1} d_{\frac{q+p+3}{2}} \overline{\frac{q+p+1}{2}} \in T-T .
\end{aligned} \\
& T \pm\left[\begin{array}{c}
\frac{p+1}{2} \\
1
\end{array}\right] \text { are neighbors of } T: \text { Finally, we consider the translated digit set } \\
& D^{\prime}=\left[\begin{array}{l}
1 \\
1
\end{array}\right]+D=\left\{\left[\begin{array}{l}
1 \\
1
\end{array}\right], d_{\frac{p+1}{2}}=\left[\begin{array}{c}
\frac{p+1}{2} \\
1
\end{array}\right], \cdots, d_{\frac{q}{2}}=\left[\begin{array}{c}
\frac{q}{2} \\
1
\end{array}\right],\left[\begin{array}{l}
1 \\
0
\end{array}\right], \cdots\left[\begin{array}{c}
\frac{q}{2} \\
0
\end{array}\right]\right\} .
\end{aligned}
$$

Then $A^{-1} d_{\frac{p+1}{2}}-A^{-2} d_{\frac{q}{2}}=0$ implies that $d_{\frac{p+1}{2}}=\overline{d_{\frac{q}{2}}}-\overline{d_{\frac{p+1}{2}}}$.

We now assume that $2|p| \leq|q+2|$ and determine candidates for possible neighbors of $T$. Considering the translated digit set

$$
D+\left[\begin{array}{l}
0 \\
1
\end{array}\right]=\left\{\left[\begin{array}{l}
0 \\
0
\end{array}\right],\left[\begin{array}{l}
1 \\
0
\end{array}\right],\left[\begin{array}{l}
2 \\
0
\end{array}\right], \cdots\left[\begin{array}{c}
\frac{q-2}{2} \\
0
\end{array}\right],\left[\begin{array}{l}
0 \\
1
\end{array}\right],\left[\begin{array}{l}
1 \\
1
\end{array}\right], \cdots\left[\frac{q-2}{2}\right]\right\},
$$

we see that any nonzero integer vector $d \in T-T$ has the form $\frac{1}{2}\left(\epsilon v+\sum_{k=1}^{\infty} A^{-k} b_{k} v\right)$, where $v=\left[\begin{array}{l}1 \\ 0\end{array}\right], \epsilon=0, \pm 1,\left|b_{k}\right| \in\{0,1,2, \ldots, q-1\}$. The proof of Theorem 4.1 in [13] shows that possible nonzero integer vectors for $\sum_{k=1}^{\infty} A^{-k} b_{k} v$ are $\pm v, \pm((p+$ 1) $v+A v), \pm(p v+A v)$. Then each nonzero $d \in T-T \subseteq \mathbb{Z}^{2}$ has one of the forms $\pm v, \pm \frac{1}{2}((p-1) v+A v), \pm \frac{1}{2}((p+1) v+A v)$, and this shows that $T$ is disk-like.

Case 2. $p$ is even: We will show that $T \pm\left[\begin{array}{l}1 \\ 0\end{array}\right], T \pm\left[\begin{array}{c}\frac{p}{2} \\ 1\end{array}\right], T \pm\left[\begin{array}{c}\frac{p+2}{2} \\ 1\end{array}\right]$ are neighbors of $T$.

$T \pm\left[\begin{array}{l}1 \\ 0\end{array}\right]$ are neighbors of $T$ : We first suppose that $p \leq q-4$. WLOG, we can study the translated digit set

$D^{\prime}=\left[\begin{array}{l}1 \\ 0\end{array}\right]+D=\left\{d_{1}=\left[\begin{array}{l}1 \\ 0\end{array}\right], \cdots, d_{\frac{q}{2}}=\left[\begin{array}{c}\frac{q}{2} \\ 0\end{array}\right], \cdots, d_{\frac{q+p}{2}}=\left[\begin{array}{c}\frac{p}{2} \\ -1\end{array}\right], \cdots,\left[\begin{array}{c}\frac{q}{2} \\ -1\end{array}\right]\right\}$.

Then $A^{-1} d_{\frac{q+p}{2}}-A^{-2} d_{\frac{q}{2}}=-d_{1}$ implies that

$$
S_{4}=\sum_{k=0}^{\infty} A^{-k}\left(A^{-1} d_{1}+A^{-2} d_{\frac{q+p}{2}}-A^{-3} d_{\frac{q}{2}}\right)=0 .
$$

Hence

$$
\begin{aligned}
-d_{1} & =A^{-1} d_{\frac{q+p}{2}}-A^{-2} d_{\frac{q}{2}}+S_{4} \\
& =A^{-1}\left[\frac{p+4}{2}\right]-A^{-1} d_{1}+\sum_{k=2}^{\infty} A^{-k}\left[\frac{p+2}{2}\right]-\sum_{k=2}^{\infty} A^{-k} d_{\frac{q}{2}} \in T-T .
\end{aligned}
$$

Next, we assume that $p=q-2$. Thus our original digit set becomes

$$
D=\left\{\left[\begin{array}{l}
0 \\
0
\end{array}\right], d_{2}=\left[\begin{array}{l}
1 \\
0
\end{array}\right], \cdots,\left[\begin{array}{c}
\frac{p}{2} \\
0
\end{array}\right],\left[\begin{array}{c}
0 \\
-1
\end{array}\right], d_{\frac{q+4}{2}}=\left[\begin{array}{c}
1 \\
-1
\end{array}\right], \cdots, d_{q}=\left[\begin{array}{c}
\frac{p}{2} \\
-1
\end{array}\right]\right\} .
$$

Using this digit set and noting that $\sum_{k=1}^{\infty} A^{-k}=(A-I)^{-1}=\left[\begin{array}{cc}p+1 & \frac{p}{2}+1 \\ 2 & 1\end{array}\right]$, we obtain

$$
A^{-1}\left((A-I)^{-1} d_{\frac{q+4}{2}}-d_{q}\right)=d_{2} \in T-T \text {. }
$$


$T \pm\left[\begin{array}{c}\frac{p+2}{2} \\ 1\end{array}\right]$ are neighbors of $T:$ WLOG, we can study the digit set $D^{\prime}=\left[\begin{array}{l}1 \\ 2\end{array}\right]+D=\left\{\left[\begin{array}{l}1 \\ 2\end{array}\right], \cdots, d_{\frac{q}{2}}=\left[\begin{array}{c}\frac{q}{2} \\ 2\end{array}\right], \cdots, d_{\frac{q+p+2}{2}}=\left[\begin{array}{c}\frac{p+2}{2} \\ 1\end{array}\right], \cdots,\left[\begin{array}{c}\frac{q}{2} \\ 1\end{array}\right]\right\}$.

Then $A^{-1} \frac{d_{q+p+2}}{2}-A^{-2} d_{\frac{q}{2}}=0$ implies that $\frac{d_{\frac{q+p+2}{2}}}{2}=\overline{d_{\frac{q}{2}}}-\overline{d_{\frac{q+p+2}{2}}} \in T-T$.

$T \pm\left[\begin{array}{c}\frac{p}{2} \\ 1\end{array}\right]$ are neighbors of $T$ : Finally, we consider the translated digit set $D^{\prime}=\left[\begin{array}{l}1 \\ 1\end{array}\right]+D=\left\{\left[\begin{array}{l}1 \\ 1\end{array}\right], \cdots, d_{\frac{p}{2}}=\left[\begin{array}{c}\frac{p}{2} \\ 1\end{array}\right], \cdots,\left[\begin{array}{c}\frac{q}{2} \\ 1\end{array}\right],\left[\begin{array}{l}1 \\ 0\end{array}\right], \cdots, d_{q}=\left[\begin{array}{c}\frac{q}{2} \\ 0\end{array}\right]\right\}$.

Then $A^{-1} d_{\frac{p}{2}}-A^{-2} d_{q}=0$ implies that $d_{\frac{p}{2}}=\overline{d_{q}}-\overline{d_{\frac{p}{2}}} \in T-T$.

We now assume that $2|p| \leq|q+2|$ and determine candidates for possible neighbors of $T$. Considering the translated digit set

$D+\left[\begin{array}{l}0 \\ 1\end{array}\right]=\left\{\left[\begin{array}{l}0 \\ 0\end{array}\right],\left[\begin{array}{l}1 \\ 0\end{array}\right],\left[\begin{array}{l}2 \\ 0\end{array}\right], \cdots,\left[\frac{q-2}{2}\right],\left[\begin{array}{l}0 \\ 1\end{array}\right],\left[\begin{array}{l}1 \\ 1\end{array}\right],\left[\begin{array}{l}2 \\ 1\end{array}\right], \cdots,\left[\frac{q-2}{2}\right]\right\}$, we see that any nonzero integer vector $d \in T-T$ has the form $\frac{1}{2}\left(\epsilon v+\sum_{k=1}^{\infty} A^{-k} b_{k} v\right)$, where $v=\left[\begin{array}{l}1 \\ 0\end{array}\right], \epsilon=0, \pm 1,\left|b_{k}\right| \in\{0,1,2, \ldots, q-1\}$. The proof of Theorem $4.1 \mathrm{in}$ 13] shows that possible nonzero integer vectors for $\sum_{k=1}^{\infty} A^{-k} b_{k} v$ are $\pm v, \pm((p+$ 1) $v+A v), \pm(p v+A v)$. Thus each nonzero $d \in T-T \subseteq \mathbb{Z}^{2}$ has one of the forms $\pm v, \pm \frac{1}{2}((p+2) v+A v), \pm \frac{1}{2}(p v+A v)$ and so $T$ is disk-like in this case.

(B) We suppose that $2|p|>|q+2|$ and prove that $T(A, D)$ is not disk-like by giving another neighbor of $T$ and using Theorem 2.2 and (A). We now consider the translated digit set

$$
\begin{aligned}
D^{\prime} & =\left[\begin{array}{c}
\frac{q}{2}+1 \\
2
\end{array}\right]+D \\
& =\left\{\left[\begin{array}{c}
\frac{q}{2}+1 \\
1
\end{array}\right], \cdots,\left[\begin{array}{l}
q \\
1
\end{array}\right],\left[\begin{array}{c}
\frac{q}{2}+1 \\
2
\end{array}\right], \cdots, d_{p+1}=\left[\begin{array}{c}
p+1 \\
2
\end{array}\right], d_{q}=\left[\begin{array}{l}
q \\
2
\end{array}\right]\right\} .
\end{aligned}
$$

Then $A^{-1} d_{q}=d_{p+1}$ and $A^{-1} d_{p+1}-A^{-2} d_{q}=0$ will imply that $d_{p+1}=\overline{d_{q}}-\overline{d_{p+1}}$. Hence $T+d_{p+1}$ is a neighbor of $T$.

\section{The ReduCiBle-CHARACTERISTIC-POLYNOMIAL CASE}

In this section, we will study a class of self-affine sets arising from expanding matrices with integer eigenvalues. A matrix $A$ is said to be unimodular if $U \in M_{n}(\mathbb{Z})$ and $|\operatorname{det}(U)|=1$. The set of such matrices will be denoted by $G L_{n}(\mathbb{Z})$.

Lemma 4.1. Suppose that the expanding matrix $A \in M_{2}(\mathbb{Z})$ has eigenvalues $m, n \in$ $\mathbb{Z}$. Then there exists a unimodular matrix $U \in G L_{2}(\mathbb{Z})$ such that

$$
A^{\prime}=U^{-1} A U=\left[\begin{array}{cc}
m & 0 \\
t & n
\end{array}\right]
$$

Proof. Choose an eigenvector $\left[\begin{array}{l}x \\ y\end{array}\right] \in \mathbb{Z}^{2}$ of $A$ corresponding to the eigenvalue $n$ such that $\operatorname{gcd}(x, y)=1$. Then there exist integers $r, s$ such that $y r-x s=1$. Now set $U=\left[\begin{array}{ll}r & x \\ s & y\end{array}\right]$. Then $U^{-1} A U$ is of the required form.

Because of Lemma 4.1, for the disk-like case, it is enough to study only the matrices $A^{\prime}$ of the form in (4.1). We have two cases in (4.1). 
(I) $t=0: T\left(A^{\prime}, D\right)$ with $D=\left\{\left[\begin{array}{l}i \\ j\end{array}\right]: 0 \leq i \leq m-1,0 \leq j \leq n-1\right\}$ is the unit square and it is disk-like. If $m<0, \quad n>0$, then $A^{\prime}=\left[\begin{array}{cc}-|m| & 0 \\ 0 & n\end{array}\right]$ and $D=$ $\left\{\left[\begin{array}{l}i \\ j\end{array}\right]: 0 \leq i \leq|m|-1,0 \leq j \leq n-1\right\}$ lead to $T\left(A^{\prime}, D\right)=\left[\frac{-|m|}{|m|+1}, \frac{1}{|m|+1}\right] \times[0,1]$, which is again a square of area 1.

Now $v=\left[\begin{array}{c}|m|-1 \\ |n|-1\end{array}\right] \in \mathbb{Z}^{2}$ gives $D=v-D$ for $D=\left\{\left[\begin{array}{l}i \\ j\end{array}\right]: 0 \leq i \leq|m|-1,0 \leq\right.$ $j \leq|n|-1\}$ so that we can make use of Proposition 2.5. To handle the other cases, namely when the expanding matrix is of the form $A^{\prime}=\left[\begin{array}{cc} \pm|m| & 0 \\ 0 & -|n|\end{array}\right]$, we use Proposition 2.5 to give a disk-like tile.

(II) $t \neq 0:$ In this case, we consider the digit set

$$
D=\left\{\left[\begin{array}{c}
i \\
t j
\end{array}\right]: 0 \leq i \leq|m|-1,0 \leq j \leq|n|-1\right\}
$$

In fact, we can make a further reduction of $A^{\prime}$ in (4.1) and $D$ in (4.2). For this, we let $B=\left[\begin{array}{ll}1 & 0 \\ 0 & t\end{array}\right]$ provided that $t \neq 0$. Then

$$
\begin{gathered}
\tilde{A}=B^{-1} A^{\prime} B=\left[\begin{array}{cc}
m & 0 \\
1 & n
\end{array}\right], \\
\tilde{D}=B^{-1} D=\left\{\left[\begin{array}{l}
i \\
j
\end{array}\right]: 0 \leq i \leq|m|-1,0 \leq j \leq|n|-1\right\} .
\end{gathered}
$$

To be able to use Theorem 2.3, we need a characterization of Haar tiles. For this characterization, the concept of a stretched tile is introduced by Lagarias and Wang [12]: $T=T(A, D)$ is called a stretched tile if $A$ and $D$ satisfy

$A=\left[\begin{array}{cc}A_{1} & 0 \\ B & A_{2}\end{array}\right], D=\left\{\left[\begin{array}{c}a_{i} \\ b_{i}\end{array}\right]+\left[\begin{array}{c}0 \\ Q c_{i j}\end{array}\right]: 1 \leq i \leq\left|\operatorname{det}\left(A_{1}\right)\right|, 1 \leq j \leq\left|\operatorname{det}\left(A_{2}\right)\right|\right\}$

where $A_{1} \in M_{r}(\mathbb{Z}), A_{2} \in M_{s}(\mathbb{Z})$ such that (i) $\left\{a_{i}\right\} \subseteq \mathbb{Z}^{r}$ is a complete residue system $\left(\bmod A_{1}\right)$; (ii) $\left\{b_{i}\right\} \subset \mathbb{Z}^{s}$; (iii) $|\operatorname{det} Q| \geq 2$ and for each $i,\left\{Q c_{i j}\right\} \subset \mathbb{Z}^{s}$ is a complete residue system $\left(\bmod A_{2}\right)$. A matrix $A$ as in this definition is called a reducible matrix. A primitive digit set $D$ is said to be standard if it is a complete residue system $(\bmod A)$. We say that two tiles $T=T(A, D)$ and $T^{\prime}=T\left(A^{\prime}, D^{\prime}\right)$ are $\mathbb{Z}$-similar if there exists a unimodular matrix $U \in G L_{n}(\mathbb{Z})$ such that $A^{\prime}=U^{-1} A U$ and $D^{\prime}=U^{-1} D$. In $[12$, it was proved that

If $T$ is a tile generated by a standard digit set and a reducible matrix, then $\mu(T)>1$ ( $\mu$ denotes the Lebesgue measure) if and only if $T$ is $\mathbb{Z}$-similar to a stretched tile.

Lemma 4.2. Let $\tilde{A}, \tilde{D}$ be as in (4.3) and (4.4) with $m=n=\lambda$. Then $T(\tilde{A}, \tilde{D})$ has Lebesgue measure 1.

Proof. By Proposition 2.5, it is enough to consider the case $m=n=\lambda>0$. Note that $\tilde{D}$ is a standard digit set. Now, we show that $T(\tilde{A}, \tilde{D})$ cannot be $\mathbb{Z}$-similar to a stretched tile and hence it is a Haar tile. $\tilde{A} U=U\left[\begin{array}{ll}x & 0 \\ y & z\end{array}\right]$ with $U=\left[\begin{array}{ll}a & b \\ c & d\end{array}\right]$, 
$U \in G L_{2}(\mathbb{Z})$, yields

$$
\begin{gathered}
\lambda a=a x+b y, \\
\lambda b=\frac{b \lambda^{2}}{x}, \\
a+\lambda c=c x+d y, \\
b+\lambda d=\frac{d \lambda^{2}}{x} .
\end{gathered}
$$

We have two cases:

a) $b \neq 0$ : Then (4.6) gives $\lambda=x$. This together with (4.8) give $b=0$, a contradiction. Thus we cannot have $b \neq 0$.

b) $b=0:|\operatorname{det}(U)|=1$ and $b=0$ imply $a d= \pm 1$. (4.5) gives $\lambda=x$. Finally, we get $y= \pm 1$ by (4.7).

Therefore, $U^{-1} \tilde{A} U=\left[\begin{array}{ll}x & 0 \\ y & z\end{array}\right]$ for a unimodular matrix $U$ if and only if $x=z=$ $\lambda, y= \pm 1$ and $U^{-1}=\left[\begin{array}{cc}\epsilon & 0 \\ c^{\prime} & \epsilon^{\prime}\end{array}\right]$, where $\epsilon, \epsilon^{\prime} \in\{ \pm 1\}$.

Now when $\left[\begin{array}{l}i \\ j\end{array}\right] \in \tilde{D}, U^{-1}\left[\begin{array}{l}i \\ j\end{array}\right]=\left[\begin{array}{c}\epsilon i \\ c^{\prime} i+\epsilon^{\prime} j\end{array}\right]$. Also $c^{\prime} i+\epsilon^{\prime} j$ cannot be of the form $b_{i}+q c_{i j}$ with $q>1$ and $c_{i j}$ are distinct for fixed $i$; for otherwise, we would have $\left|\left(c^{\prime} i+\epsilon^{\prime} 1\right)-\left(c^{\prime} i \pm 0\right)\right|=| \pm 1|=\left|q\left(c_{i 1}-c_{i 0}\right)\right|>1$. Thus $T(\tilde{A}, \tilde{D})$ cannot be $\mathbb{Z}$-similar to a stretched tile. Hence the Lebesgue measure of $T(\tilde{A}, \tilde{D})$ is 1 .

Proposition 4.3. Let $\tilde{A}, \tilde{D}$ be as in (4.3) and (4.4). Then $T(\tilde{A}, \tilde{D})$ is a Haar tile.

Proof. We first recall that $\tilde{D}$ is a standard digit set. By Lemma 4.2, we only need a proof for the case $m \neq n$. We first assume that $m, n>0$. Now, we will use the idea in the previous proof to show that $T(\tilde{A}, \tilde{D})$ is a Haar tile. Suppose that $\tilde{A} U=U\left[\begin{array}{ll}x & 0 \\ y & z\end{array}\right]$ with $U \in G L_{2}(\mathbb{Z})$. Then we get $m a=a x+b y$ and $m b=b z$. If $b=0$, then $a \neq 0$ and the first equation gives $x=m$. If $b \neq 0$, then the second equation gives $m=z$. Thus we have two cases.

Case 1. $x=m$ and $z=n$ : Then $\tilde{A} U=U\left[\begin{array}{ll}x & 0 \\ y & z\end{array}\right]$ implies $b+n d=d n$. This gives $b=0$. Therefore, $a d= \pm 1$ since $|\operatorname{det}(U)|=1$ and $U^{-1}$ has the form $\left[\begin{array}{cc}\epsilon & 0 \\ c^{\prime} & \epsilon^{\prime}\end{array}\right]$. Then $U^{-1}\left[\begin{array}{l}i \\ j\end{array}\right]=\left[\begin{array}{c}\epsilon i \\ c^{\prime} i+\epsilon^{\prime} j\end{array}\right]$. As before, $c^{\prime} i+\epsilon^{\prime} j$ cannot be of the form $b_{i}+q c_{i j}$ with $q>1$ and $c_{i j}$ are distinct for fixed $i$. Thus $T(\tilde{A}, \tilde{D})$ is a Haar tile.

Case 2. $x=n$ and $z=m: \tilde{A} U=U\left[\begin{array}{ll}x & 0 \\ y & z\end{array}\right]$ with $U=\left[\begin{array}{ll}a & b \\ c & d\end{array}\right]$ implies

$$
\begin{gathered}
a+n c=c n+d y, \\
b+n d=d m .
\end{gathered}
$$

We have two possibilities:

a) $a \neq 0$ : Then (4.9) gives $a=d y \neq 0$. By (4.10), $b=(m-n) d$. Substituting these into $|\operatorname{det}(U)|=|a d-b c|=1$ yields $d= \pm 1$. Thus $U^{-1}$ has the form $\left[\begin{array}{cc}\epsilon & \epsilon^{\prime}(m-n) \\ -c & a\end{array}\right]$, where $\epsilon, \epsilon^{\prime} \in\{ \pm 1\}$. For $\left[\begin{array}{l}i \\ j\end{array}\right] \in \tilde{D}$, we have $U^{-1}\left[\begin{array}{l}i \\ j\end{array}\right]=$ $\left[\begin{array}{c}\epsilon i+\epsilon^{\prime}(m-n) j \\ -c i+a j\end{array}\right]$. Then $U^{-1} \tilde{D}$ cannot be a digit set of a stretched tile since $m \neq n$. 
Thus $T(\tilde{A}, \tilde{D})$ cannot be $\mathbb{Z}$-similar to a stretched tile. Hence the Lebesgue measure of $T(\tilde{A}, \tilde{D})$ is 1 .

b) $a=0$ : Then $|\operatorname{det}(U)|=1$ implies $b c= \pm 1$. This together with (4.10) gives $b=(m-n) d= \pm 1$ and so $d= \pm 1 . U^{-1}$ has the form $\left[\begin{array}{cc}\epsilon & \epsilon^{\prime} \\ \epsilon^{\prime \prime} & 0\end{array}\right]$, where $\epsilon, \epsilon^{\prime}, \epsilon^{\prime \prime} \in\{ \pm 1\}$. Now when $\left[\begin{array}{l}i \\ j\end{array}\right] \in \tilde{D}, U^{-1}\left[\begin{array}{l}i \\ j\end{array}\right]=\left[\begin{array}{c}\epsilon i+\epsilon^{\prime} j \\ \epsilon^{\prime \prime} i\end{array}\right]$. Then $U^{-1} \tilde{D}$ cannot be a digit set of a stretched tile since $m \neq n$. Thus $T(\tilde{A}, \tilde{D})$ cannot be $\mathbb{Z}$-similar to a stretched tile. Hence $T$ is a Haar tile.

The proof for the case $m<0, n\rangle 0$ is similar. For the remaining two cases, we use Proposition 2.5 to finish the proof.

We can now prove the second main theorem.

The proof of Theorem 1.2, Let $\tilde{T}=T(\tilde{A}, \tilde{D})$, where $\tilde{A}$ and $\tilde{D}$ are as in Proposition 4.3. Then it suffices to show that $\tilde{T}$ is disk-like.

(A) We first assume that $m, n>0$. Let $\tilde{T} \cap\left(\tilde{T}+\left[\begin{array}{l}x \\ y\end{array}\right]\right) \neq \emptyset$. Since $\tilde{A}^{-k}$ has nonnegative entries, $|x| \leq$ the first entry of $\sum_{k=1}^{\infty} \tilde{A}^{-k}\left[\begin{array}{c}m-1 \\ \cdot\end{array}\right]=(\tilde{A}-I)^{-1}\left[\begin{array}{l}m-1 \\ \cdot\end{array}\right]=$ $\left[\begin{array}{cc}\frac{1}{m-1} & 0 \\ \frac{-1}{(m-1)(n-1)} & \frac{1}{n-1}\end{array}\right]\left[\begin{array}{c}m-1 \\ \cdot\end{array}\right]=\left[\begin{array}{l}1 \\ \cdot]\end{array}\right]$ so that $|x| \leq 1$. Also, $|y| \leq$ the second entry of $\sum_{k=1}^{\infty} \tilde{A}^{-k}\left[\begin{array}{c}0 \\ n-1\end{array}\right]=\left[\begin{array}{l}\dot{1} \\ 1\end{array}\right]$ so that $|y| \leq 1$. Thus $\left[\begin{array}{l}x \\ y\end{array}\right] \in \tilde{T}-\tilde{T}$ implies that $|x|,|y| \leq 1$. Furthermore,

$$
\begin{gathered}
{\left[\begin{array}{c}
\alpha+1 \\
0
\end{array}\right]-\left[\begin{array}{c}
\alpha \\
0
\end{array}\right]=\left[\begin{array}{l}
1 \\
0
\end{array}\right]=\sum_{k=1}^{\infty} \tilde{A}^{-k}\left[\begin{array}{c}
m-1 \\
1
\end{array}\right], 0 \leq \alpha \leq m-2,} \\
{\left[\begin{array}{c}
\beta \\
\gamma
\end{array}\right]-\left[\begin{array}{c}
\beta \\
\gamma-1
\end{array}\right]=\left[\begin{array}{c}
0 \\
1
\end{array}\right]=\sum_{k=1}^{\infty} \tilde{A}^{-k}\left[\begin{array}{c}
0 \\
n-1
\end{array}\right], 0 \leq \gamma \leq n-1,} \\
{\left[\begin{array}{c}
\alpha+1 \\
\gamma
\end{array}\right]-\left[\begin{array}{c}
\alpha \\
\gamma-1
\end{array}\right]=\left[\begin{array}{c}
1 \\
-1
\end{array}\right]=\sum_{k=1}^{\infty} \tilde{A}^{-k}\left[\begin{array}{c}
m-1 \\
1
\end{array}\right]-\sum_{k=1}^{\infty} \tilde{A}^{-k}\left[\begin{array}{c}
0 \\
n-1
\end{array}\right]}
\end{gathered}
$$

so that $\pm\left[\begin{array}{l}1 \\ 0\end{array}\right] \in \tilde{T}-\tilde{T}, \pm\left[\begin{array}{l}0 \\ 1\end{array}\right] \in \tilde{T}-\tilde{T}$, and $\pm\left[\begin{array}{c}1 \\ -1\end{array}\right] \in \tilde{T}-\tilde{T}$. We now assume that $\tilde{T} \cap\left(\tilde{T}+\left[\begin{array}{l}1 \\ y\end{array}\right]\right)$. Then $\left[\begin{array}{l}1 \\ y\end{array}\right]=\sum_{k=1}^{\infty} \tilde{A}^{-k}\left[\begin{array}{l}d_{k} \\ d_{k}^{\prime}\end{array}\right] \in \tilde{T}-\tilde{T}$ would imply that $\sum_{k=1}^{\infty} \frac{1}{m^{k}} d_{k}=1$ and hence $d_{k}=m-1$. We also note that $\tilde{A}^{-k}=\left[\begin{array}{cc}\frac{1}{m^{k}} & 0 \\ -a_{k} & \frac{1}{n^{k}}\end{array}\right]$, where $a_{k}>0$ and $\sum_{k=1}^{\infty} \tilde{A}^{-k}\left[\begin{array}{c}m-1 \\ d_{k}^{\prime}\end{array}\right] \leq\left[\begin{array}{c}1 \\ 1-(m-1) \\ \sum_{k=1}^{\infty} a_{k}\end{array}\right]$ so that $y<1$. Thus $\tilde{T}+\left[\begin{array}{l}1 \\ 1\end{array}\right]$ cannot be a neighbor of $\tilde{T}$.

Hence $\tilde{T}$ has exactly 6 neighbors: $\tilde{T} \pm\left[\begin{array}{l}1 \\ 0\end{array}\right], \tilde{T} \pm\left[\begin{array}{c}0 \\ -1\end{array}\right], \tilde{T} \pm\left(\left[\begin{array}{l}1 \\ 0\end{array}\right]+\left[\begin{array}{c}0 \\ -1\end{array}\right]\right)$. Since $\tilde{T}$ is connected by Theorem 2.1, and a Haar tile by Proposition 4.3 , it is a disk-like tile by Theorem 2.3 .

(B) For the case $m<0, n>0$, we consider the digit set $\tilde{D}+\left[\begin{array}{c}-|m| \\ 0\end{array}\right]=$ $\left\{\left[\begin{array}{l}i \\ j\end{array}\right]:-|m| \leq i \leq-1,0 \leq j \leq n-1\right\}$. Let $d_{1}=\left[\begin{array}{c}-|m| \\ 1\end{array}\right], d_{2}=\left[\begin{array}{c}-1 \\ 0\end{array}\right]$, $d_{3}=\left[\begin{array}{c}-1 \\ n-1\end{array}\right]$. We have $A^{-1} d_{1}=-d_{2}, A^{-1} d_{2}+A^{-2} d_{1}=0$ so that $\overline{d_{2} d_{1}}=0$. Then $-d_{2}=d_{1} \overline{d_{2} d_{1}}-\overline{d_{2} d_{1}} \in \tilde{T}-\tilde{T}$. Furthermore, $\left[\begin{array}{l}0 \\ 1\end{array}\right]=\overline{d_{3}}-\overline{d_{2}} \in \tilde{T}-\tilde{T}$. To see that 


$$
\begin{aligned}
& {\left[\begin{array}{c}
-1 \\
1
\end{array}\right] \in \tilde{T}-\tilde{T} \text {, we use } \tilde{D}+\left[\begin{array}{l}
1 \\
0
\end{array}\right]=\left\{\left[\begin{array}{l}
i \\
j
\end{array}\right]: 1 \leq i \leq|m|, 0 \leq j \leq n-1\right\} \text {. Then }} \\
& \tilde{A}^{-1}\left(\tilde{A}^{-1}\left[\begin{array}{l}
|m| \\
n-1
\end{array}\right]+\left[\begin{array}{l}
1 \\
0
\end{array}\right]\right)-\tilde{A}^{-1}\left[\begin{array}{c}
|m| \\
0
\end{array}\right]=\tilde{A}^{-1}\left[\begin{array}{l}
0 \\
1
\end{array}\right]-\tilde{A}^{-1}\left[\begin{array}{c}
|m| \\
0
\end{array}\right]=\left[\begin{array}{l}
1 \\
0
\end{array}\right]
\end{aligned}
$$

so that

$$
S_{5}=\tilde{A}^{-2}\left[\begin{array}{l}
1 \\
0
\end{array}\right]+\tilde{A}^{-3}\left[\begin{array}{l}
|m| \\
n-1
\end{array}\right]-\tilde{A}^{-1}\left[\begin{array}{l}
1 \\
0
\end{array}\right]-\tilde{A}^{-2}\left[\begin{array}{c}
|m| \\
0
\end{array}\right]=0 .
$$

We finally get $\left[\begin{array}{c}-1 \\ 1\end{array}\right]=\tilde{A}^{-1}\left[\begin{array}{c}|m| \\ n-1\end{array}\right]+\sum_{k=0}^{\infty} \tilde{A}^{-2 k} S_{5} \in \tilde{T}-\tilde{T}$.

To show that there are no other neighbors, we let $\left[\begin{array}{l}x \\ y\end{array}\right] \in \tilde{T}-\tilde{T}$. Then $y$ is maximized if the second entry of $\tilde{A}^{-k} d, d \in \tilde{D}-\tilde{D}$, is maximized for each $k$. This is so if $d=\left[\begin{array}{c}0 \\ n-1\end{array}\right]$ for $\tilde{A}^{-1} d$, and hence for each $\tilde{A}^{-k} d, d \in \tilde{D}-\tilde{D}$. Thus $|y| \leq$ the second entry of $\sum_{k=1}^{\infty} \tilde{A}^{-k}\left[\begin{array}{c}0 \\ n-1\end{array}\right]=\left[\begin{array}{l}0 \\ 1\end{array}\right]$. Additionally, $x \leq$ the first entry of $\sum_{k=1}^{\infty}\left(\tilde{A}^{-2 k}-\tilde{A}^{-(2 k-1)}\right)\left[\begin{array}{c}|m|-1 \\ z\end{array}\right]=\left[\begin{array}{l}1 \\ t\end{array}\right], 0 \leq|z| \leq n-1$. This shows that $|x|,|y| \leq 1$. We need to see that $\left[\begin{array}{l}1 \\ 1\end{array}\right] \notin \tilde{T}-\tilde{T}$. Notice that $t$ will reach its maximum value when $z=0$. Then $\sum_{k=1}^{\infty}\left(\tilde{A}^{-2 k}-\tilde{A}^{-(2 k-1)}\right)\left[\begin{array}{c}|m|-1 \\ 0\end{array}\right]=\left[\begin{array}{c}1 \\ -\frac{1}{n+1}\end{array}\right]$. Therefore, $\tilde{T}$ has exactly the six neighbors displayed in (A).

(C) For the remaining two cases, we use Proposition 2.5. To be able to use Proposition 2.5 for $m>0, n<0$, we need to take $\tilde{D}$ as the digit set in case (B). Then $\left[\begin{array}{c}|m|-1 \\ n-1\end{array}\right]-\tilde{D}=\tilde{D}$, and Proposition 2.5 applies. This finishes the proof.

Let $\mathcal{N}=(\tilde{T}-\tilde{T}) \cap \mathbb{Z}^{2}=\left\{ \pm\left[\begin{array}{l}1 \\ 0\end{array}\right], \pm\left[\begin{array}{l}0 \\ 1\end{array}\right], \pm\left[\begin{array}{c}1 \\ -1\end{array}\right]\right\}$. For a digit set $D$, an $r$-chain in $D$ is a finite sequence $\left\{d_{1}, \ldots, d_{r}\right\}$ of $r$ distinct vectors in $D$ such that $d_{i}-d_{i+1} \in \mathcal{N}$ for $i=1, \ldots, r-1$. We assume that the digit set $D^{\prime}$ has the following property to ensure that $T\left(\tilde{A}, D^{\prime}\right)+\mathcal{N}$ is a set of neighbors of $T\left(\tilde{A}, D^{\prime}\right)$ :

(1) if $\tilde{A}=\left[\begin{array}{cc}|m| & 0 \\ 1 & |n|\end{array}\right]$, then $D^{\prime}$ contains the digits $\left[\begin{array}{c}|m|-1 \\ 1\end{array}\right],\left[\begin{array}{c}0 \\ |n|-1\end{array}\right]$;

(2) if $\tilde{A}=\left[\begin{array}{cc}-|m| & 0 \\ 1 & |n|\end{array}\right]$, then $D^{\prime}$ involves $\left[\begin{array}{l}0 \\ 0\end{array}\right],\left[\begin{array}{l}0 \\ 1\end{array}\right],\left[\begin{array}{c}|m|-1 \\ 0\end{array}\right],\left[\begin{array}{c}|m|-1 \\ |n|-1\end{array}\right]$. Then the foregoing proof together with Theorem 2.1 also yields the following proposition, which gives a systematic way of obtaining self-affine curves. In a similar manner, we are able to generate self-affine curves in the irreducible-polynomial case.

Proposition 4.4. Let $D^{\prime}$ be a subset of $\tilde{D}$ with condition (1) or (2). Then $T\left(\tilde{A}, D^{\prime}\right)$ is a self-affine curve if and only if there is a q-chain in $D^{\prime}$, where $q=\# D^{\prime}$.

If $D^{\prime}$ is not a subset of $\tilde{D}$, but possesses property (1) or (2), then the sufficiency in Proposition 4.4 still holds. For the curve in Figure 5, the sufficiency holds even though $0 \notin D^{\prime}$.

\section{APPENDIX}

In Table 5. $A$ denotes the specified expanding integer matrix with characteristic polynomial $f(x)$ and $D$ is the associated digit set. Also $t \neq 0$ and $C$ denotes the companion matrix. We also assume that the polynomials below the second row are irreducible with $p, q>0$. In the last column, we list our results on $T(A, D)$, which 
TABLE 5

\begin{tabular}{|c|c|c|c|c|c|c|}
\hline$f(x)$ & $A$ & \multicolumn{4}{|c|}{$D$} & Disk-like \\
\hline$(x-m)(x-n)$ & $\begin{array}{cc}m & 0 \\
0 & n\end{array}$ & $\left\{\left[\begin{array}{l}i \\
j\end{array}\right.\right.$ & \multicolumn{3}{|c|}{$: 0 \leq i \leq|m|-1,0 \leq j \leq|n|-1\}$} & yes \\
\hline$(x-m)(x-n)$ & $\begin{array}{cc}m & 0 \\
t & n\end{array}$ & $\left\{\begin{array}{c}i \\
t j\end{array}\right.$ & \multicolumn{3}{|c|}{$: 0 \leq i \leq|m|-1,0 \leq j \leq|n|-1\}$} & yes \\
\hline$x^{2}-q$ & $C$ & \{ & $\begin{array}{ll}0 \\
0\end{array}$ & $\begin{array}{l}k \\
1 \\
\end{array}$ & $: 1 \leq k \leq q-1\}$ & yes \\
\hline$x^{2}+q, q$ even & $C$ & \{ & \begin{tabular}{c|c|}
$2 k$ & \\
0 &,
\end{tabular} & $\begin{array}{l}2 k \\
-1\end{array}$ & $\left.: 0 \leq k \leq \frac{q-2}{2}\right\}$ & yes \\
\hline$x^{2}+q, q$ odd & $C$ & $\left\{\begin{array}{c|c|}2 k \\
0\end{array}\right]$ & $\begin{array}{c}2 l+1 \\
-1\end{array}$ & $: 0$ & $\left.\leq k \leq \frac{q-1}{2}, 0 \leq l \leq \frac{q-3}{2}\right\}$ & yes \\
\hline $\begin{array}{l}x^{2} \pm p x+q, q \text { odd } \\
p=1 \text { or } 2 p>q+3\end{array}$ & $C$ & \{ & $\left.\begin{array}{l|l|l|}0 \\
0\end{array}\right]$, & $\begin{array}{c}k \\
\pm 1\end{array}$ & $: 1 \leq k \leq q-1\}$ & no \\
\hline $\begin{array}{l}x^{2} \pm p x+q, q \text { odd } \\
p \neq 1 \text { and } 2 p \leq q+3\end{array}$ & $C$ & $\{[$ & $\left.\begin{array}{l|l}0 \\
0\end{array}\right]$ & $\begin{array}{c}k \\
\pm 1\end{array}$ & $: 1 \leq k \leq q-1\}$ & yes \\
\hline $\begin{array}{l}x^{2} \pm p x-q, q \text { odd } \\
2 p>q-3\end{array}$ & $C$ & & $\begin{array}{l}0 \\
0\end{array}$ & $\begin{array}{c}k \\
\pm 1\end{array}$ & $: 1 \leq k \leq \mathrm{q}-1\}$ & no \\
\hline $\begin{array}{l}x^{2} \pm p x-q, q \text { odd } \\
2 p \leq q-3\end{array}$ & $C$ & \{ & $\left.\begin{array}{l|l|l|}0 \\
0\end{array}\right]$, & $\begin{array}{c}k \\
\pm 1\end{array}$ & $: 1 \leq k \leq q-1\}$ & yes \\
\hline $\begin{array}{l}x^{2}+p x \pm q, q \text { even, } \\
2 p>|q+2|\end{array}$ & $C$ & \{ & $\left.\begin{array}{c|c}2 k \\
0\end{array}\right]$, & $\begin{array}{c}2 k \\
-1\end{array}$ & $\left.: 0 \leq k \leq \frac{q-2}{2}\right\}$ & no \\
\hline $\begin{array}{l}x^{2}+p x \pm q, q \text { even } \\
2 p \leq|q+2|\end{array}$ & $C$ & \{ & $\left.\begin{array}{c|c}2 k \\
0\end{array}\right],[$ & & $\left.: 0 \leq k \leq \frac{q-2}{2}\right\}$ & yes \\
\hline
\end{tabular}
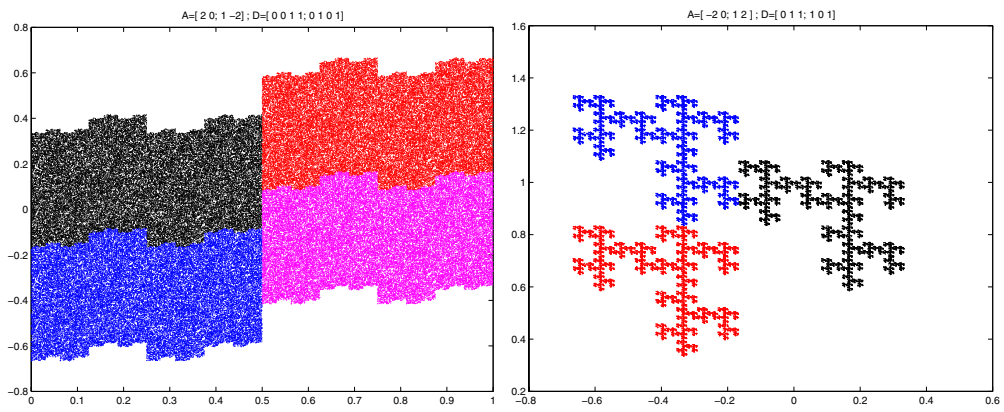

FiguRE 5. A disk-like tile of Theorem 1.2 (left) and a self-affine curve

were proved in Section 3 and Section 4 . We also note that all such $T(A, D)$ are connected tiles.

Remark. We note that the digit sets in the table are listed in a generic sense when $A$ has an irreducible characteristic polynomial. For example, $\left[\begin{array}{c}2 \\ -1\end{array}\right]$ would represent a digit of the form $2 v-A v$ for any nonzero integer vector $v$ and $C$ is the representation of an integer expanding matrix $A$ in basis $\{v, A v\}$; see Proposition 3.1.

\section{ACKNOWLEDGMENTS}

The author thanks the anonymous referees for their valuable comments and for informing him of some recent papers. Part of this work was done while the author 
was visiting the Department of Mathematics, the Chinese University of Hong Kong. He would like to thank Professor Ka-Sing Lau for his kind invitation and constant support.

\section{REFERENCES}

1. S. Akiyama and J. M. Thuswaldner, Topological properties of two-dimensional number systems, J. Theor. Nombres Bordeaux, 12 (2000), 69-79. MR.1827838(2002g:11013)

2. C. Bandt and G. Gelbrich, Classification of self-affine lattice tilings, J. London Math. Soc., 50 (1994), 581-593. MR1299459 (95g:52035)

3. C. Bandt and Y. Wang, Disk-like self-affine tiles in $\mathbb{R}^{2}$, Discrete Comput. Geom., 26 (2001), 591-601. MR1863811 (2002h:52028)

4. T. K. Dey, H. Edelsbrunner, and S. Guha, Computational topology in Advances In Discrete and Computational Geometry, B. Chazelle, J. E. Goodman and R. Pollack, eds., Contemp. Math., Vol. 223, American Mathematical Society, Providence, RI, 1999, pp. 109-143. MR1661380 (2000a:68152)

5. J. Gmainer and J. M. Thuswaldner, On disk-like self-affine tiles arising from polyominoes, Methods Appl. Anal. 13 (2006), no. 4, 351-371. MR2384259 (2009g:28028)

6. K. Gröchenig and A. Haas, Self-similar lattice tilings, J. Fourier Anal. Appl., 1 (1994), 131170. MR 1348740 (96j:52037)

7. M. Hata, On the structure of self-similar sets, Japan J. Appl. Math., 2 (1985), no. 2, pp. 381414. MR839336 (87g:58080)

8. G.T. Herman and E. Zhao, Jordan surfaces in simply connected digital spaces, J. Math. Imaging Vision, 6 (1996), 121-138. MR1390207 (97f:68199)

9. I. Kirat and K.S. Lau, On the connectedness of self-affine tiles, J. London Math. Soc. 2, 62 (2000), 291-304. MR1772188 (2001i:52027)

10. I. Kirat, K.S. Lau and H. Rao, Expanding polynomials and connectedness of self-affine tiles, Discrete and Comput. Geometry, 31 (2004), 275-286. MR2060641 (2005b:52052)

11. J. C. Lagarias and Y. Wang, Integral self-affine tiles in $\mathbb{R}^{n}$, Adv. Math., 121 (1996), 21-49. MR.1399601 (97d:52034)

12. J. C. Lagarias and Y. Wang, Integral self-affine tiles in $\mathbb{R}^{n}$. II. Lattice tilings, J. Fourier Anal. and Appl., 3 (1997), 84-102. MR1428817 (98b:52026)

13. K. S. Leung and K.S. Lau, Disklikeness of planar self-affine tiles, Trans. Amer. Math. Soc., 359 (2007), 3337-3355. MR.2299458 (2008k:52046)

14. J. Luo, Boundary local connectivity of tiles in $\mathbb{R}^{2}$, Topology Appl., 154 (2007), no. 3, 614-618. MR.2280905 (2007j:54017)

15. J. Luo, S. Akiyama and J. M. Thuswaldner, On the boundary connectedness of connected tiles, Math. Proc. Cambridge Philos. Soc., 137 (2004), no. 2, 397-410. MR2092067(2005g:37032)

16. J. Luo, H. Rao and B. Tan, Topological structure of self-similar sets, Fractals, 10 (2002), 223-227. MR 1910665 (2003d:28014)

17. S. M. Ngai and T. M. Tang. A technique in the topology of connected self-similar tiles, Fractals, 12 (2004), no.4, 389-403. MR2109984 (2006b:52018)

18. S. M. Ngai and T. M. Tang. Topology of connected self-similar tiles in the plane with disconnected interiors, Topology Appl., 150 (2005), no.1-3, 139-155. MR2133675 (2006b:52019)

19. A. Rosenfeld, Connectivity in digital pictures, J. Assoc. Comput. Mach., 17 (1970), 146-160. MR 0278576 (43:4306)

20. K. Scheicher and J. M. Thuswaldner, Neighbors of self-affine tiles in lattice tilings, Fractals in Graz, 2001, Trends Math. Birkhäuser, Basel, 2003, pp. 241-262. MR2091708 (2005f:37039)

21. R. S. Strichartz and Y. Wang, Geometry of self-affine tiles. I. Indiana Univ. Math. J. (1) 48 (1999), 1-23. MR 1722192(2000k:52017)

Department of Mathematics, Istanbul Technical University, Maslak 34469, Istanbul, TURKEY

E-mail address: ibkst@yahoo.com 